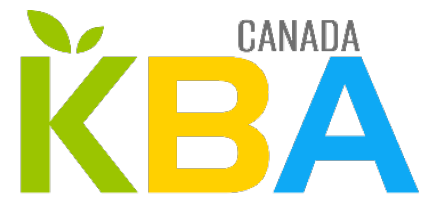

KEY BIODIVERSITY AREAS

\title{
Standard national pour l'identification des zones clés pour la biodiversité au Canada
}

Version 1.0
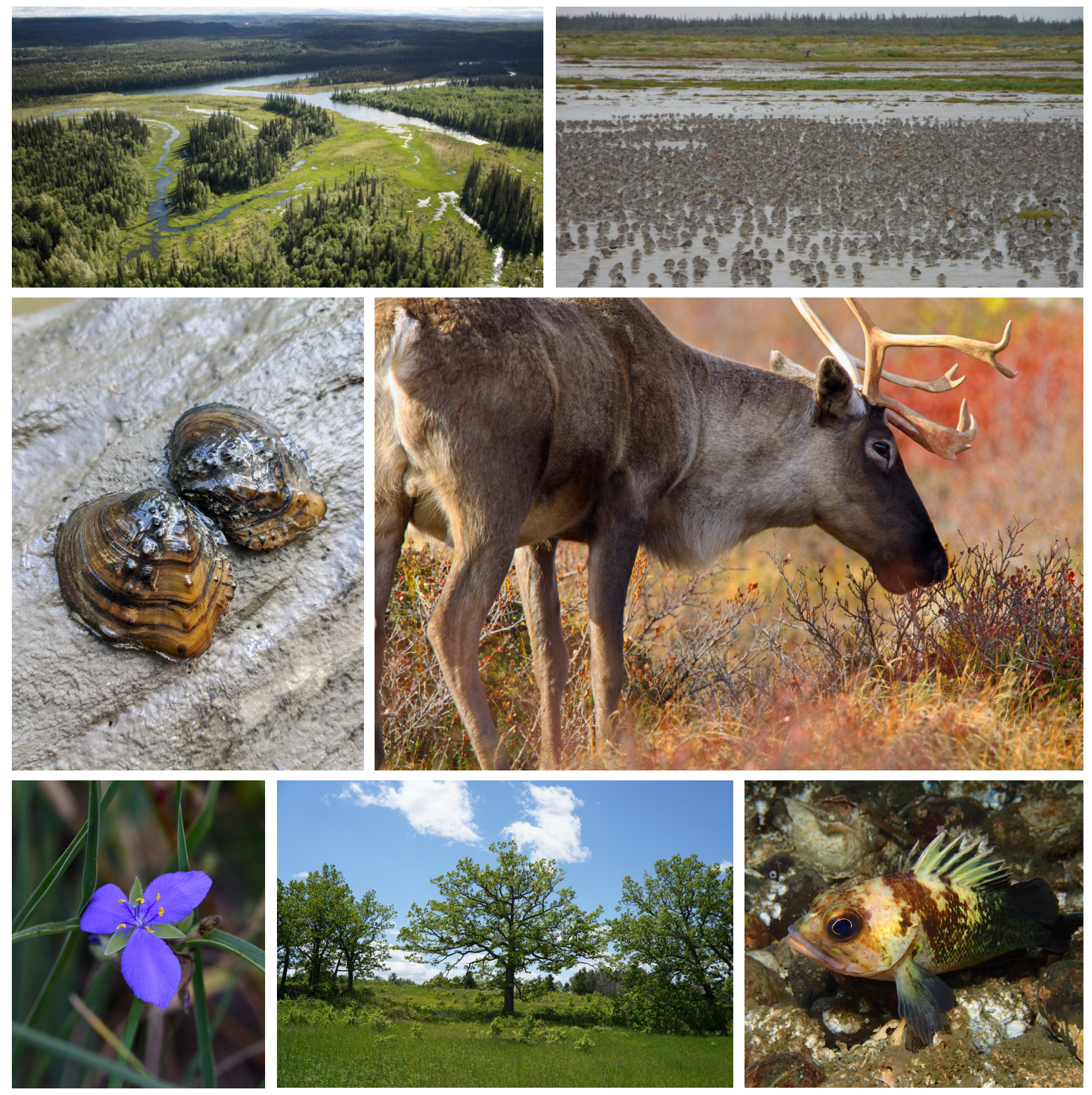


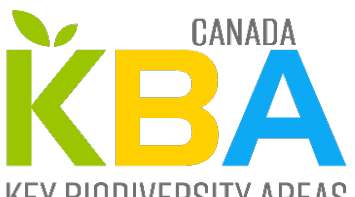

KEY BIODIVERSITY AREAS

\author{
Coalition KBA canadienne \\ kbacanada.org \\ c/o Wildlife Conservation Society Canada \\ 344 Bloor Street West, Suite 204 \\ Toronto, Ontario. M5S 3A7 \\ (416) 850-9038 \\ Renseignements généraux: craudsepp@wcs.org \\ ISBN 978-1-927895-20-7 \\ Rapport en ligne:
}

\title{
Référence
}

Coalition KBA canadienne. 2020. Standard national pour l'identification des zones clés pour la biodiversité au Canada version 1.0. Wildlife Conservation Society Canada et Key Biodiversity Area Canada Coalition, Toronto (Ontario) Canada.

\section{Photos de la page couverture:}

Les éléments de biodiversité représentés dans les photos de couverture constituent des éléments qualifiants potentiels des zones clés pour la biodiversité (Key Biodiversity Area; ou KBA employé dans le présent document) à l'échelle nationale pour une série de critères décrits dans le présent document (niveau de menace en octobre 2020).

La plaine inondable de la rivière Liard au Yukon, un écosystème d'une grande intégrité. Photo C Hilary Cooke, WCS Canada.

Les basses terres de la baie James, dans le nord-est de l'Ontario, constituent des points d'escale clés pour les oiseaux de rivage qui s'y rassemblent en grand nombre pour se reposer et s'alimenter pendant la migration. Photo (C) Allie Anderson.

Il existe deux populations de mulette feuille d'érable (Quadrula quadrula) au Canada, dont l'une est menacée à l'échelle nationale en raison de sa faible aire de répartition, de la pollution des cours d'eau et de l'établissement d'espèces envahissantes. Photo (C) Daryl Coldren, collaborateur d'iNaturalist, licence Attribution-NonCommercial 4.0 International de Creative Commons, recadrée à partir de l'original.

Le caribou (Rangifer tarandus) est un animal emblématique de la faune canadienne. Plusieurs des populations distinctes de caribous au Canada ne se trouvent nulle part ailleurs dans le monde et ont connu d'importants déclins de population. Photo (C) Susan Morse.

La tradescantie de l'Ouest (Tradescantia occidentalis) est gravement en péril au Canada, bien qu'elle soit classée comme espèce non en péril à l'échelle mondiale. À l'échelle nationale, l'espèce est restreinte à quatre habitats de dunes de sable isolés qui sont menacés par la dégradation. Photo (C) amoorehouse, collaboratrice d'iNaturalist, licence Attribution-NonCommercial 4.0 International de Creative Commons, recadrée à partir de l'original.

Les savanes de chênes des teinturiers étaient autrefois communes sur les sols sableux du sud de l'Ontario; cependant, le défrichement et la suppression des incendies ont considérablement réduit l'étendue de cet écosystème unique. Il reste peu de zones de haute qualité. Photo (C) Chelsea Marcantonio, Conservation de la Nature Canada.

Le sébaste à dos épineux (Sebastes maliger) fréquente les eaux côtières du Pacifique Nord, depuis le golfe d'Alaska jusqu'au sud de la Californie. L'espèce est gravement en péril au Canada en raison des pressions exercées par la pêche commerciale et récréative. Photo (C) amoorehouse, collaboratrice d'iNaturalist, licence Attribution-NonCommercial 4.0 International de Creative Commons, recadrée à partir de l'original. 


\title{
Standard national pour I'identification des zones clés pour la biodiversité au Canada
}

\author{
Version 1.0
}

\section{Préparé par WCS Canada avec Ciara Raudsepp-Hearne, Chloé Debyser, David Fraser et Justina C. Ray au nom de la Coalition KBA canadienne}
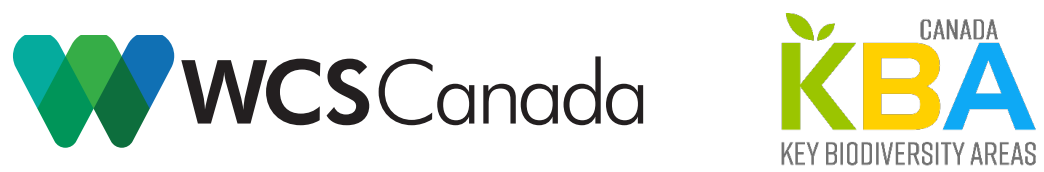

Le présent standard a été élaboré sur plusieurs années et a fait l'objet de multiples versions. Elle intègre les conseils et les informations provenant des nombreux experts et organisations mentionnés dans les remerciements ci-dessous. En outre, elle a été examinée par les agences gouvernementales fédérales, provinciales et territoriales participant au processus En route, ainsi que par 13 pairs évaluateurs.

WCS Canada coordonne le secrétariat de la Coalition KBA canadienne et a été chargé de coordonner l'élaboration de ce document. Le comité de direction de KBA Canada fournit des conseils généraux sur l'initiative de KBA Canada. Les membres de ce comité comprennent: Stephen Woodley (IUCN), Andrew Couturier (Birds Canada), Patrick Henry and Christine Terwissen (NatureServe Canada), Martin von Mirbach (WCS Canada), James Snider (WWF-Canada), Michael Bradstreet, Brenda Van Sleeuwen, and Marie-Michèle Rousseau-Clair (Nature Conservancy of Canada).

Cette version du Standard (version anglaise) a été approuvée par le Comité de Direction en février 2021. La traduction a été généreusement fournie par Environnement et Changement climatique Canada. 


\section{REMERCIEMENTS}

Nous tenons à remercier les coprésidents du Comité directeur national ${ }^{1}(\mathrm{CDN})$ du processus En route vers l'objectif 1 du Canada ${ }^{2}$ - Grant Hogg (Service canadien de la faune) et Anthony Danks (ministère de l'Environnement de la Colombie-Britannique), ainsi que Courtney Robertson (Service canadien de la faune), qui a facilité le processus de convocation des membres du CDN et leur a donné l'occasion d'examiner le document à plusieurs reprises. Zuzu Gadallah (SCF) a donné de précieux conseils et a fait appel à des experts tout au long du processus d'élaboration de ce document.

Merci à Andrew Couturier (Birds Canada) et Stephen Woodley (IUCN) pour leur contribution à la planification et à l'élaboration de ce document. Merci aux conseillers nationaux et aux coordonnateurs régionaux de KBA qui ont contribué à approfondir et mettre à l'essai l'application du Standard KBA nationale: Sherman Boates, Maria Leung, Sarah Robinson (Atlantic Canada Conservation Data Center), Kim Gauthier-Schampaert (Université de Sherbrooke), Riley Pollom, et Karin Newman. Merci à Pat Comer de NatureServe pour sa collaboration à l'application des critères des écosystèmes KBA à l'échelle nationale. Les équipes techniques de la Coalition KBA ont contribué à l'élaboration de processus et d'outils en vue d'appliquer les standards KBA nationales et mondiales au Canada : Jaime Grimm et Meg Southee (WCS Canada); Christine Terwissen, Amie Enns, Randal Greene (NatureServe Canada) et l'équipe EBAR; Dean Evans, Amanda Bichel, et Sandra Marquez (Oiseaux Canada). Jaime Grimm a également contribué à l'élaboration de la page couverture du document et de la figure 3. Nous remercions les experts en taxonomie et des sites, les collaborateurs gouvernementaux et autochtones, ainsi que les centres canadiens de données sur la conservation, pour leur aide dans le processus d'identification des KBA au Canada, qui ont permis d'élaborer du Standard KBA national.

Andy Plumptre (BirdLife International), Charlotte Boyd (UICN) et Penny Langhammer (Global Wildlife Conservation), représentant le partenariat KBA mondial, ont donné des conseils essentiels et constructifs et ont examiné attentivement le document ainsi que les précédents.

Le présent document a été préparé dans le cadre d'un contrat avec Environnement et Changement climatique Canada, qui a également apporté un soutien financier aux travaux de KBA Canada - un groupe de travail dans le cadre du processus En route vers l'objectif 1 du Canada. En outre, nous tenons sincèrement à remercier l'Alan and Patricia Koval Foundation, la Sitka Foundation, la Salamander Foundation, Suzanne Ivey Cook, la Gosling Foundation et la Fondation de la Banque Royale du Canada pour leur soutien financier.

1 https://www.conservation2020canada.ca/cdn-bios

2 https://www.conservation2020canada.ca/qui-nous-sommes 


\section{TABLE DES MATIÈRES}

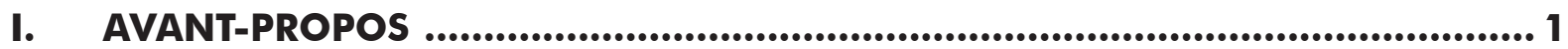

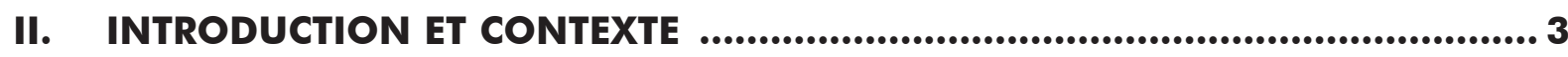

Que sont les zones clés pour la biodiversité? ............................................................................. 3

Les dimensions de la biodiversité qui répondent aux critères des KBA ..................................... 4

Un précédent au Canada pour l'adaptation nationale des standards mondiales de conservation :

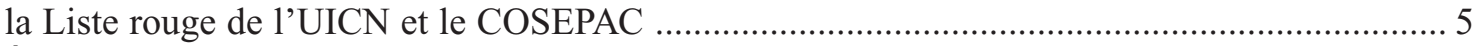

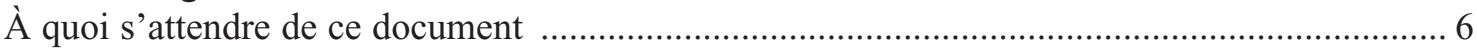

III. IDENTIFIER LES KBA MONDIALES ET NATIONALES AU CANADA .................. 6

Adaptation nationale des critères KBA mondiaux au Canada .............................................. 7

Processus d'identification des KBA mondiales et nationales au Canada ….............................. 8

IV. FACTEURS À CONSIDÉRER LORS DE L'APPLICATION DU STANDARD NATIONAL 8

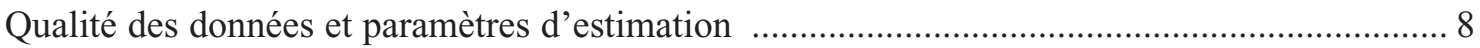

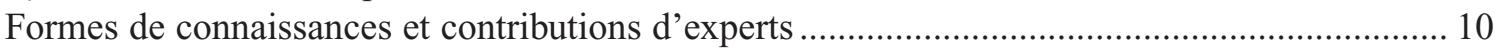

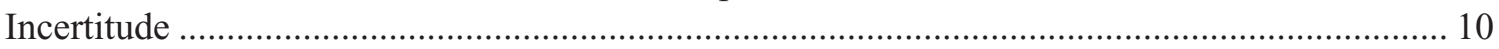

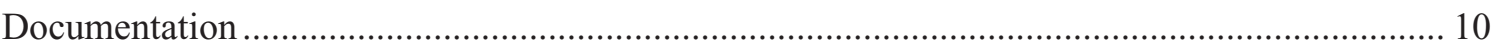

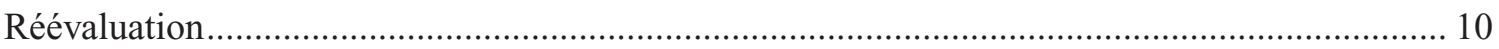

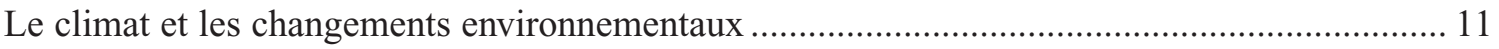

V. DÉFINITIONS ET TERMES ................................................................ 12

VI. ÉLÉMENTS DE BIODIVERSITÉ RÉPONDANT AUX CRITÈRES DE KBA NATIONALES .. 15

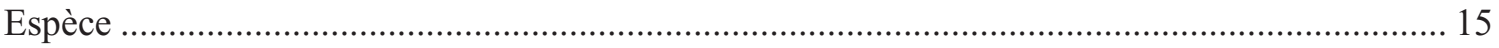

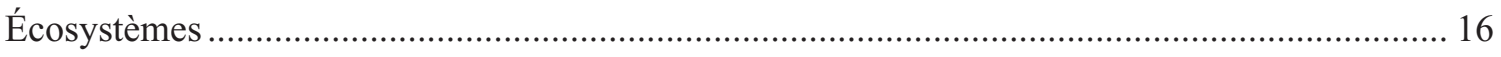

VII. CRITÈRES ET SEUILS KBA NATIONAUX ....................................................... 16

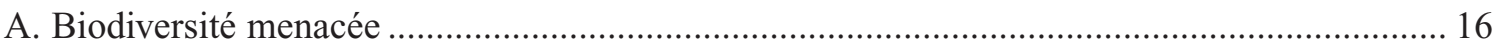

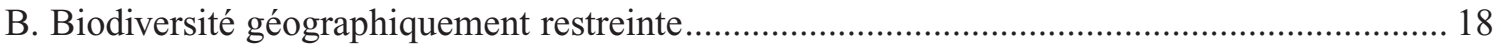

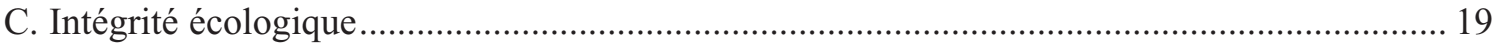

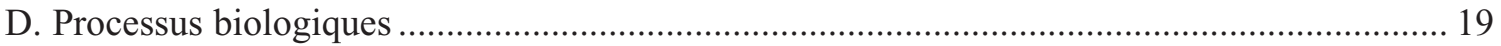

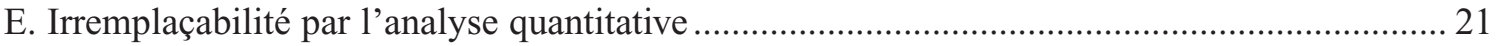

VIII. RÉSUMÉ COMPARATIF DES CRITÈRES ET SEUILS KBA NATIONAUX ET

MONDIAUX

IX. PROCÉDURES DE DÉLIMITATION AU CANADA ............................................. 25

Résumé des directives sur la délimitation tirées des Lignes directrices KBA mondiales ........... 25

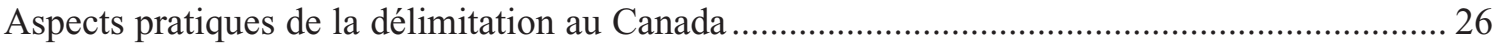

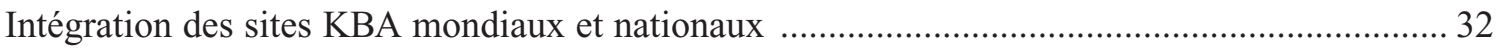

Comment élaborer des fichiers de formes KBA afin de soumettre les limites des KBA ........... 33 


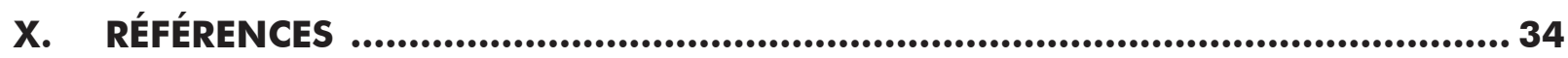

ANNEXE 1 PRÉCISIONS SUPPLÉMENTAIRES CONCERNANT LES CRITÈRES ET LES SEUILS DU STANDARD NATIONAL ....................................................................... 36

1.1 Précisions supplémentaires concernant l'application du critère $\mathrm{A} 1$....................................... 36

1.2 Utilisation de la taille de la population continentale lorsque des estimations fiables de la population nationale ne sont pas disponibles ou pertinentes (tous les critères) .......................... 38

ANNEXE 2 PROCESSUS D'EXAMEN CANADIEN ....................................................39

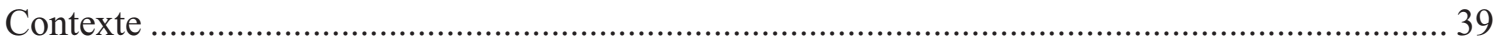

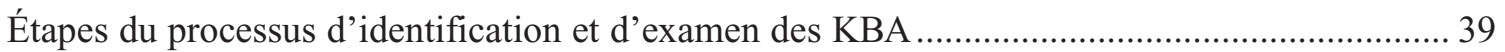




\section{AVANT-PROPOS}

Le Standard mondial pour l'identification des zones clés pour la biodiversité (UICN, 2016; ci-après, le Standard KBA mondial) a été élaboré à l'intention des groupes d'intérêt nationaux afin d'identifier les sites qui contribuent de manière significative à la conservation de la biodiversité mondiale dans les milieux terrestres, souterrains, marins et d'eau douce. Les critères KBA peuvent être appliqués aux espèces de tout groupe taxonomique ainsi qu'aux écosystèmes. L'identification objective, transparente et rigoureuse des zones de biodiversité clés permet d'acquérir des connaissances importantes sur des zones qui pourraient faire l'objet d'une intendance, d'une gestion renforcée, d'une protection officielle ou d'autres mesures de conservation.

Le Standard mondial KBA permet aux coordonnateurs nationaux des processus d'identification des KBA d'adapter les critères des KBA afin d'identifier les sites d'importance nationale, si cela est jugé utile dans le pays visé. Deuxième plus grand pays du monde, le Canada abrite un patrimoine naturel unique et riche sur un vaste territoire qui présente une grande hétérogénéité environnementale et culturelle. Bien qu'une partie de ce territoire demeure intacte sur le plan écologique, un nombre important d'espèces et d'écosystèmes sont en péril à un certain degré, en particulier dans la partie sud du pays (Kraus et Hebb, 2020). Par exemple, sur 30000 espèces marines et terrestres au Canada sur lesquelles on dispose de renseignements suffisants, $20 \%$ sont en péril à un certain degré (CCCEP, 2016), dont $68 \%$ des espèces de reptiles et $36 \%$ des espèces d'amphibiens (CCCEP, 2016). Les populations d'oiseaux de rivage, d'oiseaux de prairie et d'insectivores aériens ont diminué de $40 \%$, de $57 \%$ et de $59 \%$, respectivement, depuis les années 1970 (ICOAN-Canada, 2019). Les écosystèmes terrestres comme les milieux humides et les prairies ont connu une perte importante et continue dans la partie sud du pays (GFPTC, 2010), et entre $20 \%$ et $28 \%$ des bassins versants sont dans un état critique, selon des indices indiquant la biodiversité des poissons d'eau douce, les conditions environnementales et le stress anthropique (Chu et coll., 2015).

La perte et la dégradation des habitats étant parmi les principaux facteurs de la perte de biodiversité, on accorde de plus en plus d'attention au Canada, comme partout dans le monde, à la nécessité d'une expansion stratégique des réseaux de zones protégées et conservées, tant dans les milieux terrestres que marins. En conséquence, le Canada a pris des engagements (ECCC, 2019; Gouvernement du Canada, 2020) dans le cadre de la réalisation des objectifs mondiaux fixés par la Convention sur la diversité biologique afin d'assurer la conservation des terres et des océans. De même, les politiques du secteur privé en matière de biodiversité, les normes environnementales et les systèmes de certification suscitent de l'intérêt en vue de soutenir la planification et l'établissement des priorités en matière de conservation à différentes échelles. L'application de la méthodologie des KBA pour identifier les zones significatives sur le plan national et mondial offre une solution qui peut être mise en œuvre de manière uniforme.

Les discussions sur la mise en œuvre du Standard KBA mondial au Canada ont commencé juste après sa publication en 2016, et la Coalition KBA canadienne a été fondée en 2019 (figure 1). Le Secrétariat KBA canadien, dirigé par WCS Canada, a coordonné immédiatement la collecte des données et l'identification des KBA dans le cadre d'une série d'ateliers régionaux. Des travaux simultanés ont commencé pour adapter le Standard KBA mondial à un contexte national. Pour ce faire, un document de discussion (Fraser, 2019) a été élaboré et contient des recommandations et des justifications connexes en vue d'adapter le Standard KBA mondial à un standard national au Canada; ce standard a ensuite été mis à l'essai dans le cadre de processus régionaux d'identification des KBA. 


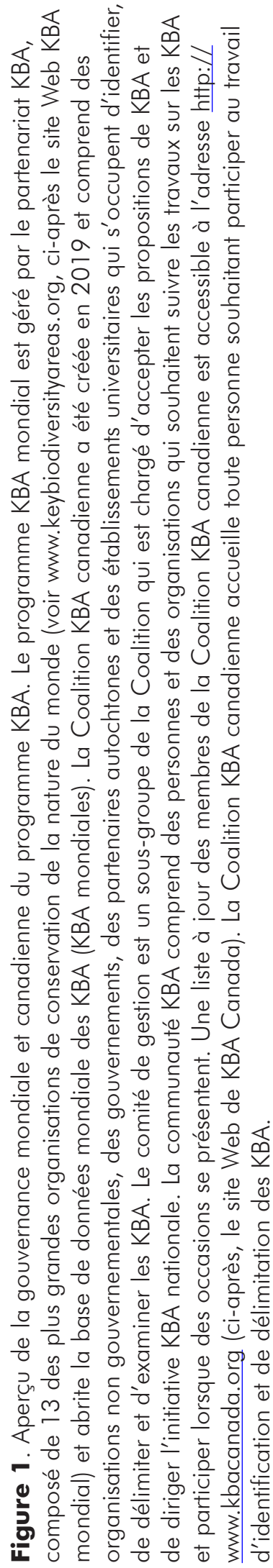
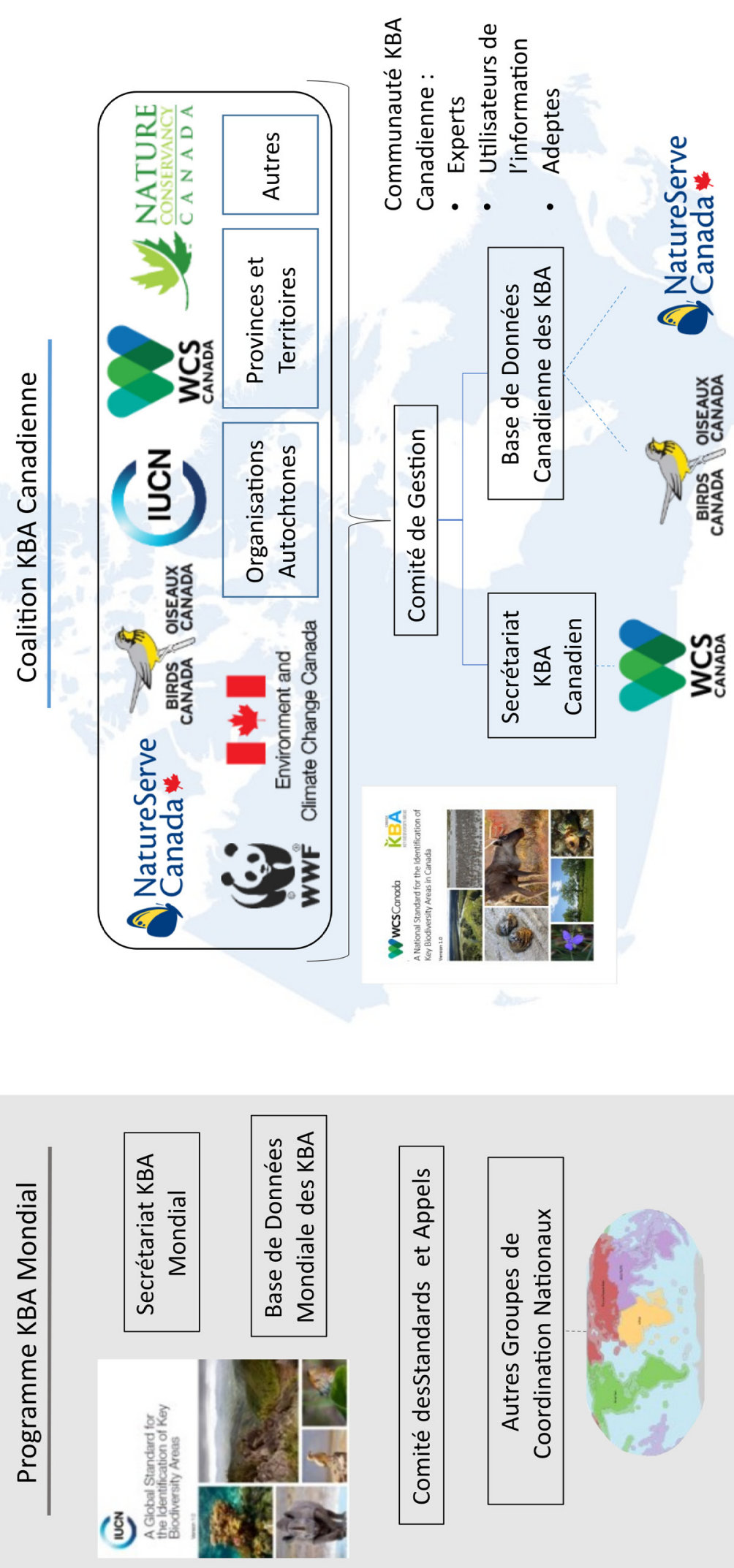
Le Canada est le premier pays à lancer la formulation d'un Standard KBA national, en se fondant sur son expérience croissante en matière d'identification des KBA. Décrite dans le présent document, le Standard KBA national pour le Canada est axé sur l'identification des KBA d'importance pour assurer la persistance de la biodiversité à l'échelle nationale. Même si ce standard est conçu comme une adaptation nationale du Standard KBA mondial, le cadre que nous avons élaboré pourrait servir de modèle à d'autres pays qui mettent en œuvre le Standard KBA à l'échelle « inframondiale », y compris à l'échelle régionale (c'est-à-dire multinationale) ailleurs dans le monde. Ce document n'a pas pour objet d'identifier les KBA au Canada en deçà de l'échelle nationale, bien que la plupart des provinces et territoires soient aussi grands que de nombreux pays en dehors de l'Amérique du Nord, ce qui rend possible la création de KBA provinciales et territoriales dans le futur.

Des lignes directrices destinées à étayer la mise en œuvre du Standard KBA mondial ont été élaborées en 2019 et révisées en 2020 (KBA Standards and Appeals Committee, 2020; ci-après «Lignes directrices KBA mondiales»). La plupart des indications contenues dans les Lignes directrices KBA mondiales s'appliquent également au Standard KBA national, bien qu'en cas de différences, le Standard KBA national doit prévaloir lorsqu'il s'agit de KBA nationales. Les Lignes directrices KBA mondiales définissent les termes clés associés à chacun des critères et expliquent comment appliquer les différents paramètres d'évaluation. Elles précisent les normes en matière de données et approfondissent les règles relatives aux éléments qualifiants permettant d'identifier une KBA. Les Lignes directrices KBA mondiales décrivent également comment déterminer les limites des KBA de manière cohérente et rigoureuse.

Outre les conseils techniques sur l'application du Standard KBA mondial, les Lignes directrices KBA mondiales énoncent des principes de collaboration avec les experts et les détenteurs de droits au cours du processus d'identification des KBA. Le processus d'identification des KBA doit être très inclusif, consultatif et ascendant. Même si toute personne disposant d'informations appropriées peut proposer un site comme KBA, il est recommandé de consulter les détenteurs de droits et les intervenants à l'échelon national et infranational (organisations non gouvernementales et gouvernementales) au cours du processus de proposition. Toutes les propositions de sites sont soumises à un examen scientifique indépendant et font l'objet d'un contrôle de cohérence de l'application du Standard KBA.

Le présent Standard KBA national comprend des directives supplémentaires concernant la délimitation des KBA (section IX) et le processus d'examen des KBA (annexe 2) qui sont pertinentes à l'application des standards nationales et mondiales au Canada. Le Secrétariat KBA canadien continuera d'élaborer des directives adaptées visant les KBA nationales et mondiales au Canada, à mesure que notre expérience s'enrichira en matière d'identification et de délimitation des KBA. À ce titre, ce document sera mis à jour dès que des renseignements supplémentaires seront disponibles au cours des processus d'identification des KBA au Canada ou que de nouvelles versions des Lignes directrices KBA mondiales seront élaborées

\section{INTRODUCTION ET CONTEXTE}

\section{Que sont les zones clés pour la biodiversité?}

Les zones clés pour la biodiversité $(\mathrm{KBA})$ sont des sites qui contribuent de manière significative à la persistance mondiale de la biodiversité. Lancée en 2016 par l'UICN et ses partenaires, le Standard KBA mondial est le résultat d'une consultation échelonnée sur 12 ans avec des experts d'organismes de conservation, de gouvernements, d'universités et du secteur privé afin de regrouper les critères et les méthodes d'identification des KBA. Le Standard KBA s'appuie sur plus de 30 ans d'expérience en matière d'identification de sites importants pour différents sous-ensembles taxonomiques, écologiques et thématiques de la biodiversité, tels que les zones importantes pour les oiseaux et la biodiversité (ZICO), les 
sites de l'Alliance for Zero Extinction (AZE) et les zones importantes pour les plantes (ZIP), qui existent tous déjà au Canada. Le Standard KBA vise à établir «des critères quantitatifs et des seuils associés pour identifier les KBA de manière objective, reproductible et transparente» (KBA Standards and Appeals Committee, 2020).

Les KBA abritent des espèces ou des écosystèmes rares ou menacés ou présentent des caractéristiques qui attirent de grands rassemblements d'animaux qui cherchent à se nourrir, se reproduire ou s'abriter. Les KBA peuvent également être des zones naturelles uniques qui ne sont pas perturbées par le développement industriel et qui comprennent des assemblages d'espèces intacts et des processus écologiques à l'état naturel. L'approche des KBA rassemble pour la première fois de nombreuses valeurs naturelles (espèces ou groupes taxonomiques, écosystèmes, etc.) sous un même toit, créant ainsi un outil fiable et quantitatif qui permet de déterminer les zones de grande valeur sur le plan de la biodiversité et d'orienter les efforts de conservation sur le terrain afin d'éviter de nouvelles pertes d'espèces et de zones sauvages. La reconnaissance d'une KBA ne conduit à aucune prescription de gestion, mais constitue une couche d'information qui peut étayer les décisions de gestion de tout type, telles que l'aménagement du territoire, la planification des zones protégées, l'intendance locale et les politiques d'utilisation des terres.

Les caractéristiques suivantes font des KBA un outil fiable et unique pour identifier les sites importants pour la biodiversité

- Les KBA respectent des seuils quantitatifs associés à un ensemble de critères;

- Les KBA ne peuvent être négociées ou rejetées s'il existe des preuves suffisantes qu'un seuil quantitatif a été atteint;

- Les KBA sont bien documentées et examinées;

- Les KBA englobent un large éventail d'éléments de biodiversité, allant des espèces macroscopiques (tous les taxons) aux écosystèmes;

- Les KBA peuvent être identifiées en fonction d'un seul ou de plusieurs éléments de biodiversité;

- Les KBA peuvent être de toute taille pouvant être considérée raisonnablement comme un «site» gérable (voir la section $V$, Définitions et termes).

Les KBA comprennent des sites individuels qui sont importants pour la persistance de certains éléments de biodiversité. Cette approche peut ne pas comprendre certains éléments de biodiversité qui sont mieux gérés à d'autres échelles (par exemple, les espèces qui sont présentes à faible densité dans le paysage et qui nécessitent une approche systémique) et, pour assurer la conservation de la biodiversité à long terme, elle doit être complétée par des facteurs tels que la connectivité, les changements climatiques, les valeurs bioculturelles ainsi que les menaces et les pressions.

\section{Les dimensions de la biodiversité qui répondent aux critères des KBA}

Les sites répondent aux critères d'une KBA s'ils atteignent des seuils quantitatifs associés à un ou plusieurs de 11 critères, regroupés en cinq principales catégories :
A. Biodiversité menacée
B. Biodiversité géographiquement restreinte
C. Intégrité écologique
D. Processus biologiques
E. Irremplaçabilité par l'analyse quantitative 
Tableau 1. Critères KBA mondiaux et éléments de biodiversité

\begin{tabular}{|c|c|c|c|}
\hline Critère & Diversité génétique & Espèces & Écosystèmes \\
\hline \multicolumn{4}{|l|}{ A. Biodiversité menacée } \\
\hline A1. Espèces menacées & $x$ & $x$ & \\
\hline A2. Types d'écosystèmes menacés & & & $x$ \\
\hline \multicolumn{4}{|l|}{ B. Biodiversité géographiquement restreinte } \\
\hline $\begin{array}{l}\text { B1. Espèces individuelles géographiquement } \\
\text { restreintes }\end{array}$ & $x$ & $x$ & \\
\hline $\begin{array}{l}\text { B2. Espèces cooccurrentes géographiquement } \\
\text { restreintes }\end{array}$ & $x$ & $x$ & \\
\hline B3. Assemblages géographiquement restreints & & $x$ & \\
\hline $\begin{array}{l}\text { B4. Types d'écosystèmes géographiquement } \\
\text { restreints }\end{array}$ & & & $x$ \\
\hline C. Intégrité écologique & & $x$ & $x$ \\
\hline \multicolumn{4}{|l|}{ D. Processus biologiques } \\
\hline D1. Agrégations démographiques & & $x$ & \\
\hline D2. Refuges écologiques & & $x$ & \\
\hline D3. Sources de recrutement & & $x$ & \\
\hline E. Irremplaçabilité par l'analyse quantitative & & $x$ & \\
\hline
\end{tabular}

Remarque : Ce tableau est adapté à partir du rapport du comité des normes et des appels KBA (2020).

Les critères et les seuils KBA mondiaux sont décrits dans le Standard KBA mondial. À partir de ces critères mondiaux, nous avons adapté les critères et les seuils KBA nationaux qui sont présentés dans ce document. Les critères KBA sont conçus expressément pour englober tous les niveaux de biodiversité, y compris la diversité génétique, les espèces et les écosystèmes (tableau 1). Ils peuvent s'appliquer aux espèces et aux écosystèmes des milieux terrestres, souterrains, d'eau douce et marins, et les seuils associés à chacun des critères peuvent être appliqués à tous les groupes taxonomiques (autres que les micro-organismes) et écosystèmes.

\section{Un précédent au Canada pour l'adaptation nationale des normes mondiales de conservation : la Liste rouge de I'UICN et le COSEPAC}

Un précédent important a été établi au Canada afin d'adapter une norme mondiale à l'échelle nationale lorsque le Comité sur la situation des espèces en péril au Canada (COSEPAC) a adapté les catégories et critères mondiaux de la Liste rouge de l'UICN (UICN, 2012) à des fins d'utilisation nationale.

Créée en 1964, la Liste rouge des espèces menacées de l'UICN est devenue le principal protocole pour évaluer la conservation mondiale des espèces en fonction de données probantes. L'application continue de cet outil a permis de mettre à l'essai et de faire évoluer considérablement la norme depuis sa création il y a plus de 50 ans. Avec le temps et l'expérience, la nécessité d'effectuer des évaluations à l'échelle internationale s'est imposée (Gärdenfors et coll., 2001; Rodríguez, 2008), étant donné l'intérêt d'établir le statut des espèces au niveau auquel elles sont gérées, d'évaluer le statut des taxons qui sont en péril à l'échelle nationale, mais qui ne le sont pas à l'échelle mondiale, d'éclairer les priorités de conservation internationales et de soutenir la protection des espèces par des lois (Mounce et coll., 2018). Pour répondre à ces besoins au Canada, le COSEPAC a été créé en 1977 à la suite d'une décision prise lors de la Conférence 
des directeurs fédéraux-provinciaux-territoriaux de la faune en vue d'élaborer « une classification nationale unique, officielle et reposant sur des données scientifiques des "espèces sauvages"3 en péril», et est devenu un organisme consultatif en vertu de la Loi fédérale sur les espèces en péril (2002) (COSEPAC, 2020a). En 1999, le COSEPAC a révisé ses critères afin d'orienter l'évaluation du statut des «espèces sauvages » à partir des catégories révisées de la Liste rouge de l'UICN (UICN, 2012) et utilise ce cadre depuis lors (Manuel des opérations et des procédures du COSEPAC).

Même si l'on peut comparer l'expérience de la nationalisation du Standard KBA avec celle du COSEPAC, il est important de souligner certaines différences entre les deux systèmes : le COSEPAC est un organisme constitué en vertu d'une loi qui, contrairement à la Coalition KBA canadienne, est mandaté par la loi pour exécuter son travail. En outre, les évaluations du COSEPAC s'appliquent aux espèces dans toute leur aire de répartition au Canada, tandis que les KBA concernent des sites bien précis. Néanmoins, l'expérience du COSEPAC au Canada constitue un modèle précieux pour adapter le Standard KBA mondial à l'échelle nationale, compte tenu de l'expérience approfondie déjà acquise en matière d'application des critères et des seuils à l'échelle canadienne

\section{À quoi s'attendre de ce document}

Le présent document constitue le Standard KBA national pour le Canada - une adaptation du Standard KBA mondial qui est pertinent au contexte canadien et qui élargit le champ d'application de l'identification des KBA dans ce pays. Le Standard KBA national comprend officiellement les définitions, les critères et les seuils connexes. Elle contient également des sections sur les procédures liées aux KBA appliquées dans un contexte canadien, telles que la délimitation et le processus d'examen des KBA au Canada. Le Standard KBA national doit être utilisée parallèlement au Standard KBA mondial et aux Lignes directrices KBA mondiales. Il ne sera peut-être pas possible de déterminer si un site répond aux critères mondiaux ou nationaux tant que les analyses ne seront pas terminées; c'est pourquoi nous présentons les critères et les seuils mondiaux parallèlement aux critères et aux seuils nationaux à la section VIIII. Les lignes directrices KBA mondiales servent à appliquer le Standard KBA national et doivent être examinées avant de procéder à l'identification et à la délimitation des KBA. Des lignes directrices supplémentaires propres au Canada figurent dans les annexes du présent document et dans d'autres publications de l'initiative KBA canadienne accessibles sur le site Web de KBA Canada.

\section{IDENTIFIER LES KBA MONDIALES ET NATIONALES AU CANADA}

Toute personne ou organisation peut utiliser le Standard KBA national et le Standard KBA mondial pour identifier les sites contribuant de manière significative à la persistance de la biodiversité dans les environnements terrestres, d'eau douce et marins au Canada. La Coalition KBA canadienne doit être tenue informée des travaux visant à identifier et à délimiter les KBA au Canada et a le pouvoir définitif de reconnaître les KBA nationales. Les propositions de KBA - qu'elles concernent des KBA nationales ou mondiales - doivent être soumises par l'intermédiaire de la Coalition KBA canadienne. Comme dans le cas du Standard KBA mondial, les critères et les seuils KBA nationaux pourraient devoir être révisés à l'avenir, en fonction de l'expérience de leur mise en œuvre.

3 Le terme «espèces sauvages», défini dans la Loi sur les espèces en péril (LEP), est équivalent à «taxon» dans le présent document (voir la section V), et figurera entre guillemets lorsqu'il sera utilisé ici. 
Figure 2. Résultats et particularités du Standard KBA national, en comparaison avec les particularités du Standard KBA mondial.

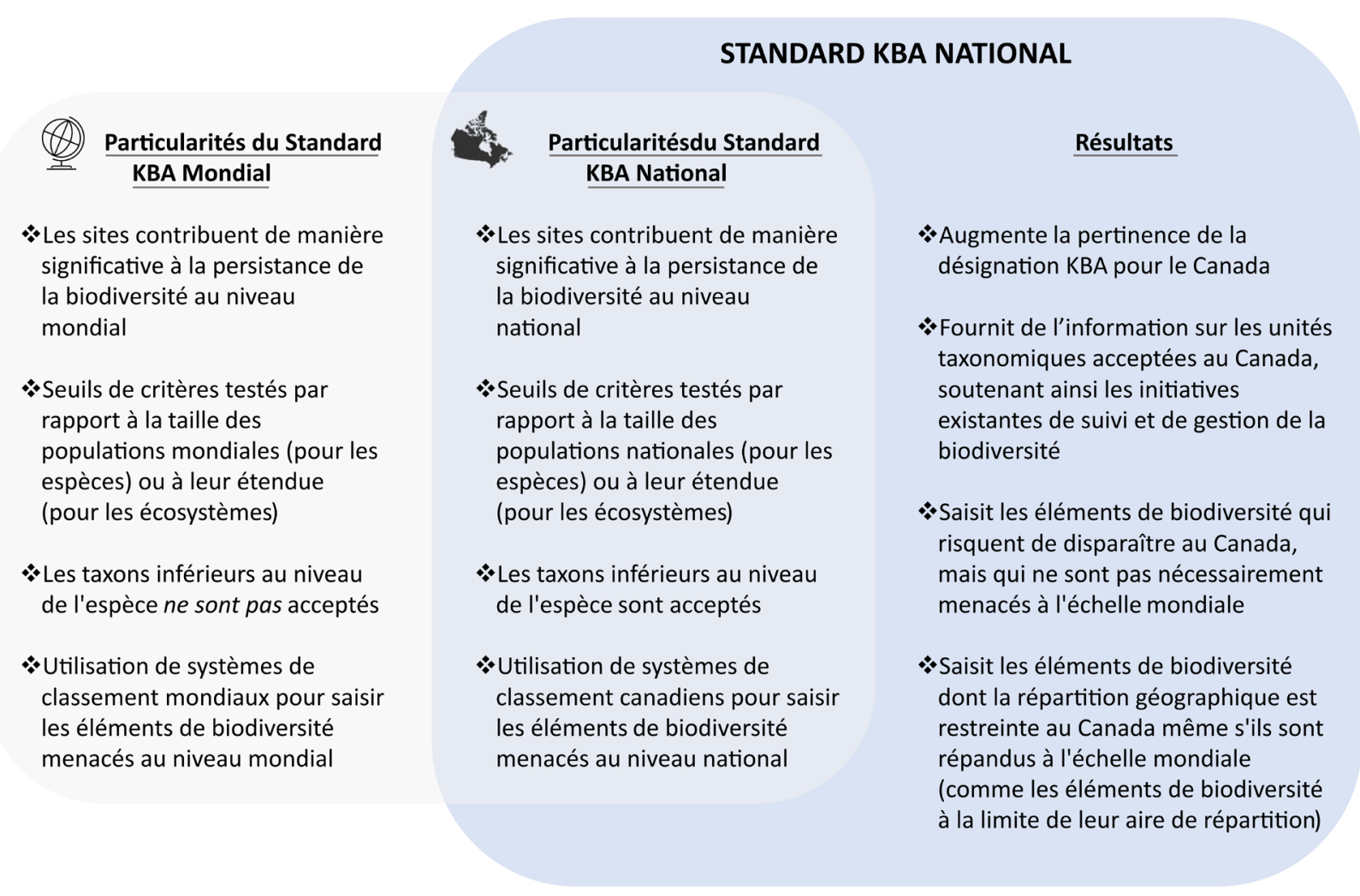

\section{Adaptation nationale des critères KBA mondiaux au Canada}

Les Standards KBA nationales et mondiales sont complémentaires, et l'identification des KBA aux deux échelons permettra de disposer de données fiables pour orienter les mesures de conservation visant à protéger la biodiversité présente sur ces sites. La figure 2 présente les principales différences entre les deux standards ainsi que les résultats visés par l'élaboration d'un Standard KBA national pour le Canada.

Les critères du Standard KBA mondial ont été adaptés pour le Standard KBA national au Canada, sans ajout. Toutefois, 4 des 11 critères ne sont pas compris dans ce Standard KBA national, à savoir :

B2, B3 - Espèces cooccurrentes géographiquement restreintes et assemblages géographiquement restreints. Ces critères ne seront pas adaptés au Standard KBA national, car ils ont été jugés non applicables à l'échelle nationale au Canada. En effet, les écosystèmes nordiques et tempérés du nord ne contiennent généralement pas d'assemblages d'espèces ayant une répartition restreinte au sein d'un groupe taxonomique, et celles qui s'y trouvent seront prises en compte par le Standard KBA mondial.

C - Intégrité écologique. Ce critère peut être adapté pour le Standard KBA national au Canada. Toutefois, l'adaptation nationale du critère $\mathrm{C}$ est reportée jusqu'à ce que des méthodes d'application de ce critère mondial aient été mises au point pour le Canada.

E - Irremplaçabilité par l'analyse quantitative. Ce critère peut être adapté pour le Standard KBA national au Canada. Toutefois, l'adaptation nationale du critère E est reportée jusqu'à ce que des méthodes d'application de ce critère mondial aient été mises au point pour le Canada. 


\section{Processus d'identification des KBA mondiales et nationales au Canada}

Le processus d'identification des KBA au Canada est le même pour les KBA mondiales et nationales. La figure 3 présente le processus d'identification des KBA en fonction des critères A, B et D. Les processus d'identification des KBA en fonction des critères $\mathrm{C}$ et $\mathrm{E}$ seront différents de ceux présentés à la figure 3 et seront élaborés lorsque ces critères seront ajoutés au Standard KBA national.

\section{FACTEURS À CONSIDÉRER LORS DE L'APPLICATION DU STANDARD NATIONAL}

Cette section décrit un certain nombre de facteurs qui contribueront à l'élaboration des projets de KBA au Canada. Une grande partie du texte est adaptée ou tirée directement du Standard KBA mondial, mais elle est reprise ici en raison de son importance pour comprendre le processus d'identification et de délimitation des KBA.

\section{Qualité des données et paramètres d'estimation}

Les critères KBA ont des seuils quantitatifs permettant de s'assurer que l'identification d'un site est transparente, objective et reproductible. Il est important de regrouper les meilleures données disponibles pour identifier les KBA. Une série de paramètres peuvent être utilisés pour estimer ou déduire si un site contient une proportion de la taille de la population mondiale ou nationale suffisante d'une espèce, ce qui démontre que la disponibilité de données fiables diffère considérablement entre les différents groupes taxonomiques et régions. Les paramètres acceptables comprennent le nombre d'individus matures, la zone d'occupation, l'étendue de l'habitat approprié, l'aire de répartition, le nombre de localités et la diversité génétique distincte. Pour évaluer les sites par rapport aux critères, il convient d'utiliser le paramètre qui permet d'obtenir les meilleures données disponibles.

Facteurs à prendre en considération pour utiliser les paramètres :

- Le nombre de localités ne doit être utilisé que lorsque l'intensité d'échantillonnage est suffisamment élevée pour présumer que les localités connues représentent correctement l'aire de répartition et la zone d'occupation des espèces.

- Plusieurs localités peuvent faire partie d'une seule KBA, et l'abondance peut varier considérablement selon les différentes localités; c'est pourquoi il n'est pas nécessairement possible de supposer qu'une espèce présente dans 100 localités ou moins atteint un seuil de $1 \%$ dans chacune de ces localités.

- En ce qui concerne les paramètres géographiques, un seuil de $1 \%$ peut généralement être déduit lorsque le site contient au moins $1 \%$ de l'étendue globale de la zone d'occupation, de l'habitat approprié ou de l'aire de répartition de l'espèce, à condition néanmoins que la présence de l'espèce soit connue sur ce site. Ces paramètres doivent être utilisés avec prudence, étant donné que les espèces ne sont pas toujours réparties de manière uniforme dans toute leur zone d'occupation ou d'habitat approprié.

- La diversité génétique se distingue des autres mesures du fait qu'elle fait référence à la proportion de la diversité génétique d'une espèce qui est présente dans une zone particulière. Un site dépassant le seuil de proportion de la diversité génétique d'une espèce peut être considéré comme une KBA (selon les critères A1, B1), même si la proportion de la population mondiale de l'espèce présente sur le site est insuffisante pour entraîner l'identification d'une KBA. L'application de la diversité génétique distincte comme paramètre d'évaluation des KBA n'a pas encore été bien vérifiée. 
Figure 3. Processus d'identification des KBA au Canada, depuis l'établissement des objectifs jusqu'à la présentation de la proposition de KBA. Le Secrétariat KBA canadien doit être consulté avant de commencer le processus d'identification des KBA et coordonnera les étapes 7 et 8 .

\section{8}

\section{Envoyer la Proposition}

a) Envoyez votre proposition de KBA au Secrétariat KBA

Canadien. Le Secrétariat examinera la validité technique de la proposition et, dans le cas d'une KBA mondiale, transmettra votre proposition au Secrétariat KBA Mondial.

b) Prévoyez une réévaluation de la KBA dans les 12 ans.

\section{7}

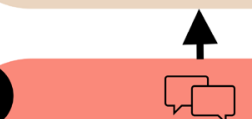

Inviter une Revue de la KBA

Avec l'aide du Secrétariat Canadien:

- Confirmez auprès des expertsque les renseignements sur le(s) élément(s) déclencheur(s) sont exacts et que les limites du site sont adéquates.

- Impliquez les parties prenantes et les détenteurs de droits pourvous assurer qu'ils sont au courant et que la proposition est complète.

\section{6}

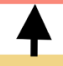

Préparer laProposition de KBA

a) Remplissez le formulaire de proposition et de nomination KBA

b) Préparez les données spatiales qui accompagneront la proposition :

- un fichier avec les limites de la KBA (obligatoire):

- des fichiers montrant l'emplacement des éléments déclencheurs au sein du site (recommandé).
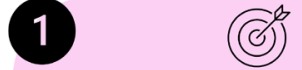

Définir l'Objectif

Vous cherchez à:

- Identifier plusieurs KBAs pour une région, un groupe taxonomique ou un critère $K B A$ ? $>$ Voir 2

- Identifier des KBAs pour un élément de biodiversité? $>$ Voir $\mathbf{3}$

- Évaluer si un site spécifique est une KBA? > Voir 4.

Dans tous les cas, contactez le Secrétariat KBA Canadien!

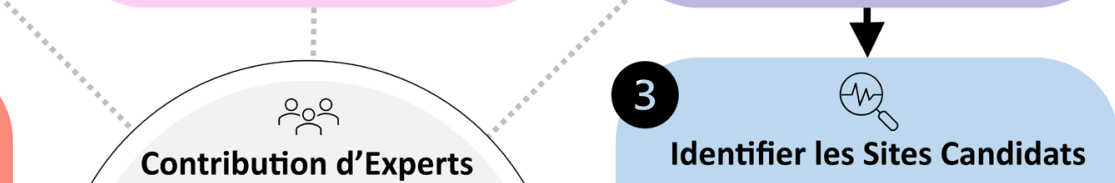

Détenteurs de connaissances autochtones et locales, ONGs, universitaires, gouvernements

Données sur la Biodiversité Évaluations de statut, données spatiales, enquêtes de terrain, littérature scientifique

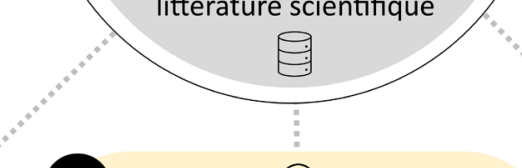

5 (O)

Délimiter la KBA

a) Pour tous les éléments répondant à un ou des critères, cartographiez où ils se trouvent au sein du site candidat.

b) Tracez la limite du site en englobant tous les éléments.

c) Harmonisez la limite avec celles d'éventuelles KBAs existantes.

d) Réajustez la limite pour faciliter la gestion du site, si nécessaire.

\section{2 \\ Lister les Éléments de Biodiv.}

a) Pour votre région, groupe taxonomique ou critère d'intérêt, dressez la liste des éléments de biodiversité susceptibles de déclencher une KBA.

b) Pour les étapes suivantes, priorisez les éléments selon leur probabilité d'atteindre les seuils des critères KBA et l'existence d'informations adéquates.

a) Pour le(s) élément(s) de biodiversité qui vous intéressent, listez les sites susceptibles de déclencher une KBA (sans les délimiter précisément à ce stade).

b) Pour les étapes suivantes, priorisez les sites selon leur probabilité d'atteindre les seuils des critères KBA et l'existence d'informations adéquates.

4 Appliquer les Critères KBA

a) Vérifiez s'il existe d'autres éléments de biodiversité surle site candidat.

b) Identifiez le(s) critère(s) KBA pertinent(s) pour chaque élément

c) Obtenez, pour chaque élément, des estimations des paramètres d'évaluation correspondants.

d) Calculez si les seuils du ou des critère(s) sont atteints. 


\section{Formes de connaissances et contributions d'experts}

Toutes les formes de connaissances servant à comprendre la répartition de la biodiversité, les écosystèmes et les paysages dans lesquels les KBA seront délimitées doivent être incluses dans les évaluations des KBA au Canada. Elles comprennent plus particulièrement les connaissances autochtones et locales. En plus des données sur la biodiversité et des informations publiées, la contribution des experts est essentielle pour appliquer adéquatement les standards KBA (mondiales et nationales). Les experts peuvent fournir des renseignements, interpréter les données, évaluer les enquêtes, évaluer la connaissance de la répartition des espèces et communiquer des connaissances sur les sites pour orienter la délimitation.

Les experts doivent participer à la fois à l'élaboration des projets de KBA et à leur examen en plusieurs étapes, notamment :

- Vérifier la validité et l'exhaustivité des données et des connaissances utilisées pour évaluer les KBA;

- Vérifier si les seuils sont atteints;

- Confirmer la pertinence écologique des limites;

- Vérifier si les critères KBA sont appliqués correctement et si les lignes directrices pour appliquer la ou les normes KBA ont été suivies;

- Fournir des informations supplémentaires sur le site de la KBA, notamment sur les caractéristiques physiques et culturelles, les mesures de conservation en cours et les menaces.

\section{Incertitude}

Les données utilisées pour évaluer si les seuils quantitatifs des critères KBA ont été atteints comportent souvent un niveau d'incertitude considérable. Cette incertitude peut résulter de la variation naturelle, de l'imprécision des termes et des définitions utilisées, du manque de données ou d'erreurs de mesure. Par exemple, les estimations de la population mondiale d'une espèce peuvent varier de plus d'un ordre de grandeur, et le nombre d'individus ou d'unités reproductrices dans un site donné peut être soumis à des variations interannuelles substantielles. Les normes relatives à la documentation pour identifier les KBA nécessitent une évaluation du niveau d'incertitude liée à l'identification et à la délimitation des KBA, tandis que la réduction progressive de cette incertitude est favorisée par la réévaluation périodique des KBA.

\section{Documentation}

L'identification d'un site comme KBA est un processus itératif qui nécessite la présence confirmée d'un ou de plusieurs éléments de biodiversité (par exemple, une espèce ou un type d'écosystème) répondant à au moins un critère KBA. Cette information doit être liée à une source fiable et être suffisamment récente pour pouvoir s'assurer que les éléments de biodiversité sont encore présents sur le site. Un minimum de données est nécessaire pour chaque KBA afin d'étayer et de justifier la reconnaissance d'un site en tant que KBA, et il est recommandé de fournir des informations détaillées supplémentaires. Les normes de documentation sont identiques pour les KBA mondiales et nationales et peuvent être téléchargées sur le site Web KBA mondial. Elles comprennent :

- Un formulaire de proposition dûment rempli;

- Un ou plusieurs fichiers de données spatiales indiquant au moins les limites de la KBA et, idéalement, les emplacements précis des différents éléments qualifiants de biodiversité. 


\section{Réévaluation}

En principe, les sites doivent être réévalués en fonction des critères et des seuils tous les 8 à 12 ans, bien que, dans la pratique, cela ne soit pas toujours possible, notamment dans le cas des sites éloignés. Des contrôles plus fréquents des KBA sont recommandés dans la mesure du possible. Les changements de statut biologique et l'évolution des connaissances peuvent influer sur le statut de KBA d'un site. Les sites qui ne répondent à aucun critère au moment de la réévaluation ne seront plus affichés comme KBA existantes, mais demeureront dans la base de données et porteront la mention «historique», «données insuffisantes» ou «doit être réévalué», selon qu'il y a lieu de croire que le site pourrait être à nouveau identifié comme KBA avec des données supplémentaires ou une enquête. La tenue d'un registre de ces KBA sera particulièrement importante dans le cas de sites éloignés difficiles à visiter (par exemple, les colonies d'oiseaux de mer) ou pour lesquels des enquêtes sont menées très rarement (par exemple, dans le Grand Nord), ou dans les cas où les espèces peuvent apparaître et disparaître en fonction des changements d'aire de répartition, des sécheresses, de la disponibilité de la nourriture, etc.

\section{Le climat et les changements environnementaux}

Les changements environnementaux résultant d'une série de facteurs de stress, tels que les changements climatiques, peuvent influer sur la biodiversité d'une KBA à un point tel que le site ne répond plus aux critères d'une KBA; cela sera déterminé au moment de la réévaluation. Il est également possible qu'une KBA gagne en importance à la suite d'un changement climatique ou que de nouveaux sites soient reconnus. La réévaluation des sites tous les 8 à 12 ans s'avère donc importante pour conserver des données précises au fil du temps.

Il est souhaitable de prévoir les répercussions à court terme des changements climatiques et d'autres facteurs de stress environnementaux, comme la destruction de l'habitat, la pollution et les espèces envahissantes, et de réaliser des analyses de vulnérabilité sur les sites. Cependant, une prédiction indiquant qu'un site est vulnérable aux changements climatiques ou à tout autre changement environnemental ne doit pas empêcher sa reconnaissance en tant que KBA. Lorsque les possibilités de gestion et la complexité topographique le permettent (par exemple dans les systèmes de montagne qui permettent un mouvement en altitude), la délimitation du site peut comprendre des habitats refuges ou des zones convenables pour l'adaptation à court terme des espèces et des écosystèmes en péril. Cela ne doit être réalisé que sur les sites où les données permettent de démontrer la pertinence de cette approche.

Il est possible de prévoir l'emplacement futur des KBA dans le cadre des scénarios de changement climatique. Ces modèles prédictifs seront importants pour planifier les actions de conservation à l'échelle nationale et régionale. Cependant, les KBA ne peuvent être identifiées que sur la base de la présence actuelle des éléments de biodiversité pour lesquels elles sont importantes, plutôt que sur des répartitions futures prévues. 


\section{DÉFINITIONS ET TERMES}

Cette section contient les termes essentiels qui s'appliquent à la mise en application du Standard KBA national. Les définitions utilisées qui sont les mêmes que dans le Standard KBA mondial sont marquées d'un astérisque et sont tirées directement de celle-ci. Cette liste de termes n'est pas exhaustive, et des définitions supplémentaires figurent dans le Standard KBA mondial et les Lignes directrices.

\section{Agrégation (critère D)*}

Un regroupement géographiquement restreint d'individus qui a généralement lieu lors d'un stade de vie précis, comme les périodes de reproduction, d'alimentation ou de migration. Ce regroupement est indiqué par une abondance relative très localisée, de deux ou plusieurs ordres de grandeur supérieurs au nombre d'individus ou à la densité moyenne de l'espèce au cours de ses autres stades de vie. Les agrégations se caractérisent par le fait que la concentration d'une proportion importante de la population d'une espèce dans l'espace et le temps augmente la vulnérabilité de l'espèce à l'exploitation ou à d'autres menaces.

\section{Aire de répartition (critère $A, B$ )*}

Les limites actuelles connues de répartition d'une espèce, représentant tous les sites d'occurrence connus, déduits ou prévus (UICN, 2012), y compris les translocations à but de conservation en dehors de l'habitat naturel (IUCN Standards and Petitions Subcommittee, 2014), mais ne comprenant pas le vagabondage (espèces observées une fois ou de façon sporadique, mais connues pour ne pas être originaires de la région).

\section{Catégorie de statut du COSEPAC}

Le COSEPAC procède à une évaluation du risque de disparition du pays ou de la planète d'une «espèce sauvage » au Canada et à une désignation ultérieure - le registre des résultats de l'évaluation du COSEPAC (COSEPAC, 2020b). Les catégories VD (en voie de disparition) et M (menacée) du COSEPAC sont applicables au Standard KBA national.

\section{COSEPAC}

Le Comité sur la situation des espèces en péril au Canada (COSEPAC) est un comité consultatif indépendant qui agit auprès du ministre fédéral de l'Environnement et du Changement climatique et qui se réunit deux fois par année pour évaluer la situation des «espèces sauvages» menacées de disparition. Ses membres, des experts de la biologie des espèces sauvages provenant du milieu universitaire, de la fonction publique, d'organisations non gouvernementales et du secteur privé, sont chargés d'identifier les espèces sauvages qui risquent de disparaître du Canada.

\section{Élément qualifiant (Critères A-E)*}

Élément de biodiversité (par exemple une espèce ou un écosystème) pour lequel au moins un critère de KBA et le seuil associé sont atteints.

\section{Endémique*}

Une espèce ayant une aire de répartition mondiale entièrement limitée à une zone géographique définie comme une région, un pays ou un site. 


\section{Espèce}

Dans le Standard KBA mondial, les seuils associés aux critères fondés sur les espèces (c'est-à-dire A1, B13, D13, E) sont conçus pour être appliqués au niveau de l'espèce. Les sous-espèces, les unités significatives du point de vue de l'évolution ou les variétés ne peuvent entraîner une désignation de KBA mondiale (sauf si un site remplit les conditions requises parce qu'il comporte une proportion seuil de diversité génétique distincte dans une espèce. Voir le critère A1 du Standard KBA mondial). La Standard KBA national s'applique aux espèces et taxons sous le niveau de l'espèce.

\section{Espèce sauvage}

Les espèces sauvages définies dans la Loi sur les espèces en péril (et par le COSEPAC) comme «espèce, sous-espèce, variété ou population géographiquement ou génétiquement distincte d'animal, de plante ou d'un autre organisme d'origine sauvage (sauf une bactérie ou un virus) qui est soit indigène du Canada ou qui s'est propagée au Canada sans intervention humaine et y est présente depuis au moins cinquante ans » (COSEPAC, 2019). Équivalent à « taxon » employé dans le présent document.

\section{Étendue de l'habitat approprié (Critères A, B)*}

La zone réunissant des conditions écologiques potentiellement convenables, telles que les types de végétation ou de substrats incluant les préférences en matière d'altitude ou de profondeur, de température et d'humidité, d'une espèce donnée (Beresford et coll., 2011).

\section{Géographiquement restreint (Critère B)*}

Un élément de biodiversité ayant une répartition mondiale restreinte, mesurée par l'aire ou l'étendue de l'habitat approprié ou la zone d'occupation, et donc en grande partie confiné ou endémique à une partie relativement petite de la planète telle qu'une biorégion, une écorégion ou un site.

\section{Individus matures (critères A, B)*}

Le nombre d'individus connus, estimés ou présumés capables de se reproduire comme défini par l'UICN (2012).

\section{Taxon infraspécifique}

Une unité taxonomique sous le niveau de l'espèce, y compris les sous-espèces, les variétés, les populations et les unités désignables.

\section{Intégrité écologique (Critère C)*}

Une condition qui maintient des assemblages d'espèces et des processus écologiques intacts, dans leur état naturel, par rapport à un point de repère historique approprié, et qui est caractérisée par un habitat naturel contigu ayant une perturbation anthropique industrielle directe minimale.

\section{Liste rouge des écosystèmes (critère A2)}

La Liste rouge des catégories et critères des écosystèmes de l'UICN est une norme mondiale pour évaluer l'état des écosystèmes, applicable à l'échelle locale, nationale, régionale et mondiale.

\section{Liste rouge des espèces menacées (critère $\mathrm{A1}$ )}

La Liste rouge des espèces menacées de l'UICN consiste en un inventaire complet de l'état de conservation mondial des espèces végétales et animales. Elle repose sur un ensemble de critères quantitatifs pour évaluer le risque de disparition de milliers d'espèces. Les catégories CR, EN et VU de l'UICN peuvent être utilisées dans le cadre du Standard KBA mondial. 


\section{Localité (critères A, B)*}

Une localité d'échantillonnage est un point indiqué par des coordonnées précises de latitude et de longitude. Notez que le terme «localité», tel que défini ici, est fondamentalement et conceptuellement différent du terme «localité» utilisé dans les catégories et critères de la Liste rouge de l'UICN (UICN, 2012).

\section{Rangs de NatureServe: Rang G et rang N (critère A)}

Le classement du statut de conservation d'un taxon à l'échelle mondiale $(\mathrm{G})$ et nationale $(\mathrm{N})$ attribué par NatureServe (Faber-Langendoen et coll., 2012).

\section{Seuil (critères A, B, D)*}

Chiffre ou pourcentage minimum qui détermine si la présence d'un élément de biodiversité sur un site est suffisamment importante pour que le site soit considéré comme une KBA en vertu d'un critère ou souscritère donné.

\section{Site}

Une zone géographique sur la terre et/ou dans l'eau comportant des frontières écologiques, physiques, administratives ou de gestion. Dans le contexte des KBA, il n'y a pas de taille minimale ou maximale pour un site, mais celui-ci doit être de taille «gérable». Dans le contexte de KBA, les termes «site» et «zone» sont utilisés de manière interchangeable.

\section{Taille de la population (critères $\mathrm{A}, \mathrm{B}, \mathrm{D})$}

Le nombre total, au niveau mondial, d'individus matures d'une espèce (UICN, 2012a). La «taille de la population» est utilisée dans le Standard KBA national plutôt que le terme "population», que l'UICN (2012) utilise pour désigner le nombre total d'individus d'une espèce. La taille de la population mondiale est définie comme le nombre total d'individus matures sur Terre.

\section{Taille de la population nationale (critères $\mathrm{A}, \mathrm{B}, \mathrm{D})$}

La taille de la population nationale désigne le nombre total d'individus matures au Canada. Divers paramètres peuvent être utilisés pour estimer la taille de la population, y compris des paramètres fondés sur la superficie comme l'aire de répartition, la zone d'occupation, etc. (voir la section VIII). En ce qui concerne les espèces migratrices, les oiseaux plus particulièrement, la taille de la population continentale est souvent plus pertinente que la taille de la population nationale et peut être utilisée dans les évaluations des KBA nationales (voir l'annexe 1).

\section{Taxon}

Une espèce ou taxon infraspécifique (par exemple, une unité désignable).

\section{Type d'écosystème (critères A, B)}

Une unité d'écosystème définie pour une évaluation standard et reproductible. Elle est définie par un ensemble particulier de variables liées à son biote indigène caractéristique, son environnement abiotique ou complexe, les interactions au sein et entre eux, et un espace physique dans lequel ceux-ci fonctionnent (Keith et coll., 2013). Les critères KBA doivent être appliqués de manière cohérente aux unités à un niveau déterminé dans une hiérarchie de classification des écosystèmes uniforme au niveau mondial ou national (p. ex., Faber-Langendoen et coll., 2014). 


\section{Unité désignable (UD) (critères A, B, D)}

Entités taxonomiques sous le niveau de l'espèce évaluées par le COSEPAC. Les unités désignables sont définies comme étant «distinctes et importantes dans l'évolution taxonomique d'une espèce». Par «important», on entend une unité qui est significative pour l'héritage évolutif d'une espèce et dont la perte ne pourrait probablement pas être compensée par une dispersion naturelle (COSEPAC, 2018).

\section{Unité reproductrice (critères $A, B$ )*}

Le nombre minimum et la combinaison d'individus matures nécessaires pour déclencher un événement reproducteur réussi sur un site (Eisenberg, 1977). Des exemples de cinq unités reproductrices comprennent cinq paires, cinq femelles reproductrices dans un harem, et cinq individus reproducteurs d'une espèce végétale.

\section{Zone d'occupation (critère A, B)*}

La zone d'occupation effective d'une espèce est la superficie effectivement occupée par une espèce dans son aire de distribution (UICN 2012a).

\section{VI. ÉLÉMENTS DE BIODIVERSITÉ RÉPONDANT AUX CRITÈRES DE KBA NATIONALES}

\section{Espèces}

Les critères de KBA nationales axés sur les espèces (A1, B1 et D1-3) peuvent être appliqués à la fois aux espèces et aux sous-espèces (collectivement appelées «taxons») ${ }^{4}$. Les taxons admissibles doivent être reconnus comme des unités taxonomiques au Canada. Les rapports d'évaluation et de situation du COSEPAC constituent la source principale de données taxonomiques validées pour le Canada. En l'absence d'évaluation ou de rapport de situation du COSEPAC pour un taxon, d'autres systèmes taxonomiques peuvent être utilisés, notamment celui adopté par NatureServe Canada (NatureServe, 2018). Des preuves doivent pouvoir démontrer que les taxons infraspécifiques sont distincts et significatifs (COSEPAC, 2018).

En outre, un taxon ne peut constituer un élément qualifiant de KBA nationale au Canada s'il présente l'une des caractéristiques suivantes :

- Disparu;

- Disparu ou absent du Canada, et non réintroduit;

- Présent au Canada uniquement de manière accidentelle ou irrégulière;

- Exotique au Canada;

- Un hybride.

4 Cette approche diffère de celle des critères mondiaux A1, B1 et D1-D3, qui sont appliqués aux espèces uniquement. 


\section{Écosystèmes}

Les écosystèmes du Canada n'ont pas été cartographiés de manière cohérente à l'échelle nationale, mais différents outils à l'échelle provinciale et nationale peuvent être adaptés pour effectuer une évaluation des KBA, notamment la Classification nationale de la végétation canadienne, qui est incomplète, ainsi que les classifications provinciales des écosystèmes et de la végétation. Tout doit être mis en œuvre pour utiliser les systèmes de classification nationaux ou régionaux (écosystèmes/végétation) en place, le cas échéant, de manière à assurer la cohérence avec les normes de définition et les conventions méthodologiques canadiennes; nous prévoyons que les versions futures de cette norme comprendront ces détails. Nous prévoyons aussi que des directives plus détaillées seront élaborées en matière d'application des critères KBA aux écosystèmes à l'échelle nationale en fonction de l'expérience acquise.

Pour entraîner un processus d'identification de KBA national au Canada, un type d'écosystème doit être cartographié à l'équivalent du niveau 5 du système de classification à six niveaux de la Liste rouge des écosystèmes (ci-après dénommée Typologie de la Liste rouge des écosystèmes) (Keith et coll., 2020). Cette mesure correspond approximativement aux niveaux des groupes ou des alliances de la classification internationale de la végétation (CIV) et de la Classification nationale de la végétation canadienne (CNVC) (Faber-Langendoen et coll., 2018; Baldwin et coll., 2019). En ce qui concerne les évaluations des KBA, le niveau des groupes de la CIV ou de la CNCV doit être utilisé pour les écosystèmes non forestiers; pour les écosystèmes forestiers, c'est le niveau des alliances qui doit être utilisé (résultat de l'atelier d'experts KBA, février 2019, Ottawa).

\section{CRITÈRES ET SEUILS KBA NATIONAUX}

Cette section contient les caractéristiques de chacun des sept critères du Standard KBA national. Bon nombre des critères et des seuils sont identiques à ceux du Standard KBA mondial, à la seule différence que le critère est appliqué à l'échelle du Canada (c'est-à-dire à la taille des populations nationales ou à l'étendue des écosystèmes par opposition à la taille et à l'étendue des populations mondiales) et peut être appliqué aux taxons sous le niveau de l'espèce. Toutefois, on autorise que les chiffres de population continentale ou biogéographique puissent être utilisés pour les espèces qui traversent les frontières internationales (par exemple les oiseaux; l'annexe 1 contient des détails supplémentaires sur les exceptions et les cas rares).

\section{A. Biodiversité menacée}

\section{A1. Espèces menacées}

Les sites classés comme KBA nationales selon le critère A1 comptent une proportion importante de la population nationale d'un taxon qui risque fortement de disparaître au Canada, et contribuent ainsi à la persistance nationale de la biodiversité sur le plan génétique et taxonomique.

Le site comporte régulièrement une ou plusieurs des caractéristiques suivantes :

a) $\geq 0,5 \%$ de la taille de la population nationale $E T \geq 5$ unités reproductrices d'un taxon menacé à l'échelle nationale 5 au niveau 1 ;

b) $\geq 1 \%$ de la taille de la population nationale $E T \geq 10$ unités reproductrices d'un taxon menacé à l'échelle nationale au niveau 2;

e) En réalité, la taille totale de la population nationale d'un taxon menacé au niveau 1 OU la zone où un taxon probablement disparu ou probablement disparu au Canada est le plus susceptible d'être présent au Canada. ${ }^{6}$ 
Les espèces migratrices peuvent répondre au critère A1 dans leur aire de reproduction et de nonreproduction (voir l'annexe 1.1.3); dans les sites de non-reproduction, le seuil des unités reproductrices peut être interprété comme le nombre d'individus matures.

\section{Taxons menacés à l'échelle nationale}

Un taxon est considéré comme menacé à l'échelle nationale aux niveaux suivants :

- Niveau 1 : s'il a un statut «en voie de disparition» (VD) du COSEPAC ou un rang N "gravement en péril» (N1) de NatureServe. Si le taxon est endémique au Canada, une catégorie «en danger critique» (CR) ou «en danger» (EN) de l'UICN, ou un rang G «gravement en péril» (G1 ou T1), qualifierait également le taxon comme menacé à l'échelle nationale au niveau 1.

- Niveau 2 : s'il a un statut «menacé» $(\mathrm{M})$ du COSEPAC ou un rang N «en péril» (N2) de NatureServe. Si le taxon est endémique au Canada, une catégorie «vulnérable» (VU) de l'UICN, ou un rang G «en péril» (G2 ou T2), qualifierait également le taxon comme menacé à l'échelle nationale au niveau 2.

L'annexe 1 contient des détails sur la classification précise des taxons ayant deux ou plusieurs rangs contradictoires.

En règle générale, seules les catégories de statut du COSEPAC, les rangs $\mathrm{N}$ et les rangs $\mathrm{G}$ datant de moins de 12 ans, ainsi que les catégories de l'UICN attribuées il y a moins de 10 ans, doivent être prises en compte pour évaluer si un taxon est menacé à l'échelle nationale afin de garantir l'utilisation des informations les plus récentes. Les exceptions à cette règle doivent être déterminées au cas par cas, notamment lorsque les mesures de contrôle sont insuffisantes ou que les réévaluations sont retardées. En outre, si le taxon a reçu un rang $\mathrm{N}$ qui fait partie d'une échelle (par exemple N1N3), il convient d'utiliser l'option la plus prudente (c'est-à-dire N1) ${ }^{7}$.

\section{Proportion de la taille de la population nationale d'un taxon}

La proportion de la taille de la population nationale peut être observée ou déduite selon l'un des critères suivants :

(i) nombre d'individus matures;

(ii) zone d'occupation;

(iii) étendue de l'habitat approprié;

(iv) aire de répartition;

(v) nombre de localités;

(vi) diversité génétique distincte.

5 Dans de rares cas, les taxons qui sont menacés à l'échelle mondiale peuvent également entraîner un processus d'identification de KBA nationale conformément au critère A1 (tous les sous-critères). Pour plus de détails, voir l'annexe 1.1.2.

6 L'identification d'une KBA nationale pour un taxon qui est possiblement disparu du pays ou de la planète n'est autorisée que conformément au sous-critère A1e, dans les rares cas où il est justifié de croire que le taxon est toujours présent sur le site bien qu'il n'ait pas été repéré.

7 Ce traitement des rangs diffère de celui des Lignes directrices KBA mondiales, qui prévoient que le rang arrondi doit être conservé pour les évaluations mondiales selon le critère A1. Dans le cas des évaluations KBA nationales, une approche prudente permettra de s'assurer que tous les taxons menacés sont pris en compte selon le critère A1. L'utilisation du rang le plus prudent s'applique également aux rangs $\mathrm{G}$ qualifiant les espèces endémiques canadiennes comme étant menacées à l'échelle nationale au Canada. 


\section{A2. Types d'écosystèmes menacés}

Les sites qui sont admissibles comme KBA nationales selon le critère A2 détiennent une proportion importante de l'étendue nationale d'un type d'écosystème confronté à un risque élevé d'effondrement à l'échelle mondiale, et contribuent ainsi à la persistance nationale et mondiale de la biodiversité des écosystèmes.

Le site comporte habituellement une ou plusieurs des caractéristiques suivantes :

a) $\geq 5 \%$ de l'étendue nationale d'un type d'écosystème CR ou EN à l'échelle mondiale;

b) $\geq 10 \%$ de l'étendue nationale d'un type d'écosystème VU à l'échelle mondiale.

Les types d'écosystèmes menacés comprennent les écosystèmes évalués comme étant en danger critique (CR), en danger (EN) ou vulnérables (VU) à l'échelle mondiale selon la Liste rouge des catégories et critères des écosystèmes de l'UICN (UICN 2015) dans la hiérarchie de classification des écosystèmes décrite à la section $\mathrm{VI}^{8}$.

\section{B. Biodiversité géographiquement restreinte}

\section{B1. Espèces individuelles géographiquement restreintes}

Les sites identifiés comme KBA nationales selon le critère B1 comptent une proportion importante de la taille de la population nationale d'un taxon et contribuent ainsi de manière significative à la persistance nationale de la biodiversité sur le plan génétique et taxonomique.

Le site abrite régulièrement $\geq 10 \%$ de la taille de la population nationale $E T \geq 10$ unités reproductrices d'un taxon.

Le critère B1 se distingue du critère D1 par l'occurrence régulière de tous les stades de la vie d'un taxon sur un site.

\section{Proportion de la taille de la population nationale d'un taxon}

La proportion de la taille de la population nationale peut être observée ou déduite selon l'un des critères suivants :

(i) nombre d'individus matures;

(ii) zone d'occupation;

(iii) étendue de l'habitat approprié;

(iv) aire de répartition;

(v) nombre de localités;

(vi) diversité génétique distincte.

8 Les évaluations des menaces pesant sur les écosystèmes à l'échelle nationale pourront être acceptées à l'avenir, compte tenu de l'expérience acquise en matière d'application du critère A2. 


\section{B2. Espèces cooccurrentes géographiquement restreintes}

Le critère B2 des KBA ne sera pas adapté pour le Standard KBA national. En effet, les écosystèmes nordiques et tempérés du nord ont tendance à ne pas abriter d'assemblages d'espèces ayant une aire de répartition restreinte au sein d'un même groupe taxonomique, et le critère est donc moins pertinent pour le Canada. Veuillez consulter le Standard KBA mondial pour obtenir des informations sur l'identification des KBA mondiales en fonction du critère B2.

\section{B3. Assemblages géographiquement restreints}

Le critère B3 des KBA ne sera pas adapté pour la Standard KBA national. En effet, les écosystèmes nordiques et tempérés du nord ont tendance à ne pas abriter d'assemblages d'espèces ayant une aire de répartition restreinte au sein d'un même groupe taxonomique, et le critère est donc moins pertinent pour le Canada. Veuillez consulter le Standard KBA mondial pour obtenir des informations sur l'identification des KBA mondiales en fonction du critère B3.

\section{B4. Types d'écosystèmes géographiquement restreints}

Les sites identifiés comme KBA nationales selon le critère B4 comptent une partie importante de l'étendue nationale d'un type d'écosystème et contribuent ainsi de manière significative à la persistance nationale de la biodiversité des écosystèmes.

Le site comprend $\geq 20 \%$ de l'étendue nationale d'un type d'écosystème.

Les types d'écosystèmes examinés figurent dans la classification des écosystèmes décrite à la section VI.

\section{Intégrité écologique}

Le critère C du Standard KBA mondial sera adapté pour le Standard KBA national au Canada. Toutefois, cette adaptation est reportée jusqu'à ce que des méthodes d'application du critère $\mathrm{C}$ du Standard KBA mondial soient mises au point pour le Canada.

\section{Processus biologiques}

\section{D1. Agrégations démographiques}

Les sites classés comme KBA nationales selon le critère D1 comptent une proportion importante de la taille de la population nationale d'un taxon au cours d'un ou plusieurs stades de vie, et contribuent ainsi de manière significative à la persistance nationale de la biodiversité sur le plan taxonomique.

Le site comporte régulièrement une ou plusieurs des caractéristiques suivantes :

a) Une agrégation représentant $\geq 1 \%$ de la taille de la population nationale d'un taxon, sur une saison, et pendant un ou plusieurs stades clés de son cycle de vie;

b) Un certain nombre d'individus matures permettant de classer le site parmi les 10 plus grandes agrégations du taxon connues au Canada. 
Les agrégations se produisent généralement au moment de la reproduction, de l'alimentation ou de la migration et se caractérisent par une abondance relative très localisée, supérieure de deux ou plusieurs ordres de grandeur aux nombres ou densités moyens enregistrés par le taxon à d'autres stades de son cycle de vie. Un site est considéré comme abritant un taxon de façon «prévisible» si celui-ci est reconnu comme ayant été présent sur le site pendant au moins deux tiers des années durant lesquelles des données adéquates sont accessibles pour la saison concernée (par exemple, la saison de reproduction dans le cas d'un agrégat de reproduction); le nombre total d'années examinées ne doit pas être inférieur à trois.

Le critère D1 n'est pas destiné à identifier les sites qui abritent tous les stades clés du cycle de vie d'un taxon; ces sites peuvent être identifiés au titre des critères A1 ou B1. Le concept d'agrégation est toutefois suffisamment général pour inclure les taxons qui demeurent regroupés pendant la plupart ou la totalité de leur cycle de vie lorsqu'ils se déplacent d'un site à l'autre. Dans le cas du sous-critère D1b, le seuil s'applique à toutes les fonctions du cycle de vie plutôt qu'à des fonctions précises (p. ex., la reproduction ou l'alimentation). Une agrégation ne peut satisfaire au critère D1b si l'on sait qu'elle ne satisfait pas au critère D1a (c'est-à-dire que ce critère ne doit être appliqué qu'aux agrégations qui comprennent au moins $1 \%$ de la population de l'espèce, mais pour lesquelles il n'existe pas suffisamment de données pour vérifier l'atteinte du seuil). Le long des corridors migratoires, les KBA doivent être identifiées pour les sites d'escale ou les goulots d'étranglement plutôt que pour l'ensemble du corridor.

\section{Proportion de la taille de la population nationale d'un taxon}

La proportion de la taille de la population nationale peut être observée ou déduite selon le critère suivant :

(i) nombre d'individus matures.

\section{D2. Refuges écologiques}

Les sites classés comme KBA nationales selon le critère D2 abritent une proportion importante de la population nationale d'un taxon pendant les périodes de stress environnemental et contribuent ainsi de manière significative à la persistance de la biodiversité des taxons.

Le site contient $\geq 10 \%$ de la population nationale d'un ou plusieurs taxons pendant les périodes de stress environnemental, pour lesquelles l'évidence historique montre que ce site a servi de refuge dans le passé et pour lequel il existe des preuves qui suggèrent qu'il continuerait de le faire dans un avenir prévisible.

Les taxons, à tous les stades de leur vie, peuvent se concentrer dans ces sites qui maintiennent les ressources nécessaires, comme la nourriture et l'eau, pendant les périodes de stress environnemental, lorsque les conditions ailleurs deviennent inhospitalières. Ces changements temporaires dans les conditions climatiques ou écologiques, sur une échelle de temps de plusieurs années ou décennies, comme lors de graves sécheresses, peuvent amener les individus d'un taxon à se concentrer durant ces périodes sur des sites particuliers. Cette période plus longue permet de distinguer les refuges écologiques des agrégations démographiques et géographiques décrites dans le critère D1.

\section{Proportion de la taille de la population nationale d'un taxon}

La proportion de la taille de la population nationale peut être observée ou déduite selon le critère suivant :

(i) nombre d'individus'.

9 Ce critère diffère du critère D2 de le Standard KBA mondial, qui prévoit que la taille de la population doit être observée à partir du nombre d'individus matures. Le changement de paramètre pour le «nombre d'individus» dans le Standard KBA national permettra aux KBA nationales selon le critère D2 d'abriter des refuges 


\section{D3. Sources de recrutement}

Les sites classés comme KBA nationales selon le critère D3 sont des sites qui produisent une proportion significative de la taille de la population nationale d'un taxon et qui contribuent ainsi de manière significative à la persistance de la biodiversité mondiale des espèces.

Le site produit de manière prévisible des propagules, des larves ou des juvéniles qui maintiennent $\geq 10 \%$ de la population nationale d'un taxon.

Contrairement aux sites identifiés selon les critères D1 et D2, dans lesquels les individus d'un taxon sont présents dans un site dans des proportions significatives à l'échelle nationale, mais à des échelles de temps différentes, le critère D3 s'applique aux taxons dont les individus se dispersent en dehors du site dans des proportions significatives à l'échelle nationale. Ces sources contribuent largement au recrutement d'un taxon ailleurs, même si le nombre d'individus matures sur le site peut être faible ou nul. Par conséquent, le seuil est applicable à la taille de la population nationale adulte présente en grande partie en dehors du site, plutôt qu'au nombre d'individus immatures dans le site.

\section{Proportion de la taille de la population nationale d'un taxon}

La proportion de la taille de la population nationale peut être observée ou déduite selon le critère suivant :

(i) nombre d'individus matures.

\section{E. Irremplaçabilité par analyse quantitative}

Ce critère du Standard KBA mondial peut être adapté pour le Standard KBA national au Canada. Toutefois, cette adaptation est reportée jusqu'à ce que des méthodes d'application du critère E du Standard KBA mondial soient mises au point et mises à l'essai.

\section{RÉSUMÉ COMPARATIF DES CRITÈRES ET SEUILS KBA NATIONAUX ET MONDIAUX}

Les tableaux suivants présentent un résumé des critères et des seuils KBA nationaux. Des renseignements sur les critères et les seuils KBA mondiaux sont également inclus à des fins de comparaison. Des détails et des définitions supplémentaires figurent dans le Standard KBA mondial et les Lignes directrices KBA mondiales. Dans tous les tableaux, le seuil de population ou d'étendue s'applique à la taille de la population (pour les espèces) ou à l'étendue (pour les écosystèmes) à l'échelle mondiale dans le cas des KBA mondiales, et à la taille de la population ou à l'étendue à l'échelle nationale dans le cas des KBA nationales. L'exigence relative aux unités reproductrices est identique pour les KBA mondiales et nationales. 


\section{A. Biodiversité menacée}

\begin{tabular}{|c|c|c|c|c|}
\hline \multirow{2}{*}{$\begin{array}{l}\text { Sous- } \\
\text { critère }\end{array}$} & \multicolumn{2}{|c|}{ Élément de biodiversité qualifiant } & \multirow{2}{*}{$\begin{array}{l}\text { Seuil de pop. } \\
\text { ou d'étendue }\end{array}$} & \multirow{2}{*}{$\begin{array}{l}\text { Unités } \\
\text { reproductrices }\end{array}$} \\
\hline & KBA mondiale & KBA nationale & & \\
\hline \multicolumn{5}{|c|}{ A1. Espèces menacées } \\
\hline a & Espèces CR ou EN & $\begin{array}{l}\text { Taxon menacé à l'échelle } \\
\text { nationale } 10 \text { au niveau } 1\end{array}$ & $0,5 \%$ & 5 \\
\hline$b$ & Espèces VU & $\begin{array}{l}\text { Taxon menacé à l'échelle } \\
\text { nationale au niveau } 2\end{array}$ & $1 \%$ & 10 \\
\hline C & $\begin{array}{l}\text { Espèces évaluées comme } \\
\text { CR ou EN uniquement en } \\
\text { raison de la réduction de la } \\
\text { taille de la population dans } \\
\text { le passé ou le présent }\end{array}$ & & $0,1 \%$ & 5 \\
\hline$d$ & $\begin{array}{l}\text { Espèces évaluées comme } \\
\text { VU uniquement en raison } \\
\text { de la réduction de la taille } \\
\text { de la population dans le } \\
\text { passé ou le présent }\end{array}$ & & $0,2 \%$ & 10 \\
\hline & Espèces CR ou EN & $\begin{array}{l}\text { Taxon menacé à l'échelle } \\
\text { nationale au niveau } 1\end{array}$ & Toute la population & - \\
\hline e & $\begin{array}{l}\text { Site où une espèce CR(PE) } \\
\text { ou CR(PEW) est la plus } \\
\text { susceptible d'être présente } \\
\text { à l'échelle mondiale }\end{array}$ & $\begin{array}{l}\text { Site où un taxon } \\
\text { probablement disparu du } \\
\text { pays ou de la planète est } \\
\text { le plus susceptible de se } \\
\text { trouver au Canada }\end{array}$ & - & - \\
\hline \multicolumn{5}{|c|}{ A2. Types d'écosystèmes menacés } \\
\hline a & $\begin{array}{l}\text { Type d'écosystème CR ou } \\
\text { EN }\end{array}$ & $\begin{array}{l}\text { Type d'écosystème CR ou } \\
\text { EN }\end{array}$ & $5 \%$ & - \\
\hline$b$ & Type d'écosystème VU & Type d'écosystème VU & $10 \%$ & - \\
\hline
\end{tabular}

10 Dans de rares cas, les taxons qui sont menacés à l'échelle mondiale peuvent également entraîner un processus d'identification de KBA nationale conformément au critère A1 (sous-critères a, b ou e). Voir l'annexe 1 pour en savoir plus. 
B. Biodiversité géographiquement restreinte

\begin{tabular}{|c|c|c|c|c|c|}
\hline \multirow{2}{*}{$\begin{array}{l}\text { Sous- } \\
\text { critère }\end{array}$} & \multicolumn{3}{|c|}{ Élément de biodiversité qualifiant } & \multirow{2}{*}{$\begin{array}{c}\text { Seuil de pop. ou } \\
\text { d'étendue }\end{array}$} & \multirow{2}{*}{$\begin{array}{l}\text { Unités } \\
\text { reproductrices }\end{array}$} \\
\hline & KBA mondiale & \multicolumn{2}{|c|}{ KBA nationale } & & \\
\hline \multicolumn{6}{|c|}{ B1. Espèces individuelles géographiquement restreintes } \\
\hline- & Toutes les espèces & \multicolumn{2}{|c|}{ Tous les taxons } & $10 \%$ & 10 \\
\hline \multicolumn{6}{|c|}{ B2. Espèces cooccurrentes géographiquement restreintes } \\
\hline- & $\begin{array}{l}\text { Plusieurs espèces ayant } \\
\text { une aire de répartition } \\
\text { restreinte et appartenant } \\
\text { au même groupe } \\
\text { taxonomique : soit } \\
\geq 2 \text { espèces OU } 0,02 \% \\
\text { du nombre mondial } \\
\text { d'espèces du groupe } \\
\text { taxonomique, la valeur la } \\
\text { plus élevée étant retenue. }\end{array}$ & 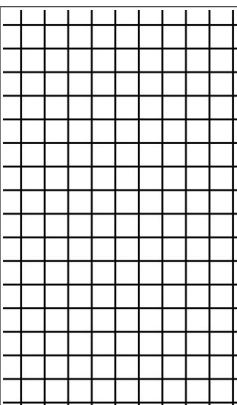 & +7 & $1 \%$ & - \\
\hline
\end{tabular}

B3. Assemblages géographiquement restreints

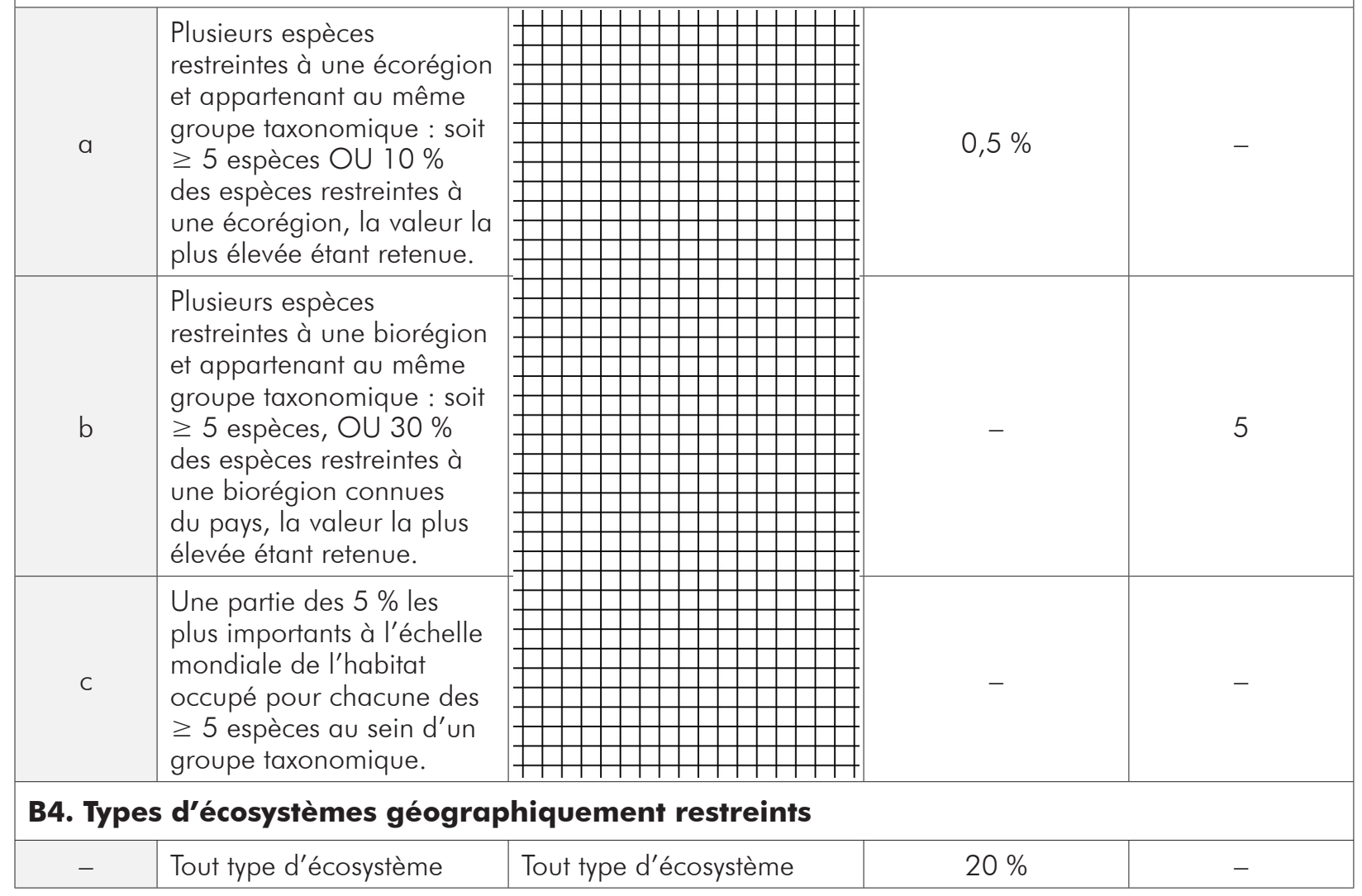




\section{Intégrité écologique}

\begin{tabular}{|c|c|c|c|c|}
\hline \multirow{2}{*}{$\begin{array}{l}\text { Sous- } \\
\text { critère }\end{array}$} & \multicolumn{2}{|c|}{ Élément de biodiversité qualifiant } & \multirow{2}{*}{$\begin{array}{l}\text { Seuil de pop. ou } \\
\text { d'étendue }\end{array}$} & \multirow{2}{*}{$\begin{array}{l}\text { Unités } \\
\text { reproductrices }\end{array}$} \\
\hline & KBA mondiale & KBA nationale & & \\
\hline- & $\begin{array}{l}\text { Un des } \leq 2 \text { sites par } \\
\text { écorégion caractérisée } \\
\text { par des communautés } \\
\text { écologiques totalement } \\
\text { intactes. }\end{array}$ & $\begin{array}{l}\text { F Reporté sous réserve } \\
\text { F de l'élaboration de la } \\
\text { E méthodologie }\end{array}$ & - & - \\
\hline
\end{tabular}

\section{Processus biologiques}

\begin{tabular}{|c|c|c|c|c|}
\hline \multirow{2}{*}{$\begin{array}{l}\text { Sous- } \\
\text { critère }\end{array}$} & \multicolumn{2}{|c|}{ Élément de biodiversité qualifiant } & \multirow{2}{*}{$\begin{array}{c}\text { Seuil de pop. ou } \\
\text { d'étendue }\end{array}$} & \multirow{2}{*}{$\begin{array}{l}\text { Unités } \\
\text { reproductrices }\end{array}$} \\
\hline & KBA mondiale & KBA nationale & & \\
\hline \multicolumn{5}{|c|}{ D1. Agrégations démographiques } \\
\hline a & $\begin{array}{l}\text { Agrégation d'une espèce } \\
\text { au cours d'une saison et } \\
\text { pendant un ou plusieurs } \\
\text { stades clés de son cycle } \\
\text { de vie }\end{array}$ & $\begin{array}{l}\text { Agrégation d'un taxon sur } \\
\text { une saison et pendant un } \\
\text { ou plusieurs stades clés de } \\
\text { son cycle de vie }\end{array}$ & $1 \%$ & - \\
\hline$b$ & $\begin{array}{l}\text { Une des } 10 \text { plus grandes } \\
\text { agrégations connues pour } \\
\text { une espèce dans le monde }\end{array}$ & $\begin{array}{l}\text { Une des } 10 \text { plus grandes } \\
\text { agrégations connues pour } \\
\text { un taxon au Canada }\end{array}$ & - & - \\
\hline \multicolumn{5}{|c|}{ D2. Refuges écologiques } \\
\hline- & $\begin{array}{l}\text { Agrégation d'individus } \\
\text { matures d'une espèce } \\
\text { pendant des périodes de } \\
\text { stress environnemental } \\
\text { passées, présentes ou } \\
\text { futures }\end{array}$ & $\begin{array}{l}\text { Agrégation des individus } \\
\text { d'un taxon au cours } \\
\text { de périodes de stress } \\
\text { environnemental passées, } \\
\text { présentes ou futures }\end{array}$ & $10 \%$ & - \\
\hline \multicolumn{5}{|c|}{ D3. Sources de recrutement } \\
\hline- & $\begin{array}{l}\text { Propagules, larves ou } \\
\text { juvéniles maintenant } \\
\geq 10 \% \text { de la taille de } \\
\text { la population mondiale } \\
\text { d'une espèce }\end{array}$ & $\begin{array}{l}\text { Propagules, larves ou } \\
\text { juvéniles maintenant } \\
\geq 10 \% \text { de la taille de la } \\
\text { population mondiale d'un } \\
\text { taxon }\end{array}$ & - & - \\
\hline
\end{tabular}

\section{E. Irremplaçabilité par l'analyse quantitative}

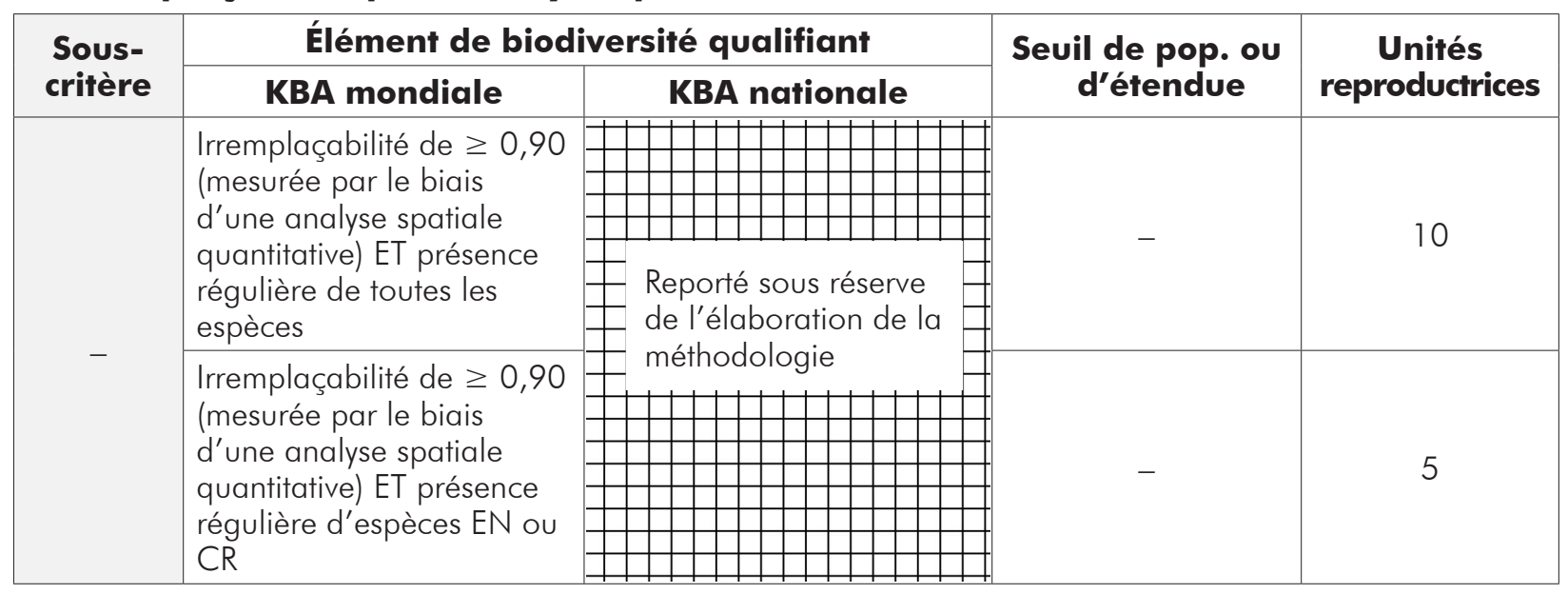




\section{PROCÉDURES DE DÉLIMITATION AU CANADA}

La délimitation a pour but de déterminer les limites d'un site qui sont pertinentes sur le plan écologique et qui constituent une base pour d'éventuelles activités de gestion. Plus précisément, l'objectif consiste à créer les meilleures conditions pour favoriser la persistance des éléments de biodiversité pour lesquels le site est important, en fonction de leurs exigences écologiques et du contexte socioculturel, économique et de gestion, dans la mesure où le site définitif délimité respecte le seuil d'au moins un critère KBA.

Pour délimiter une KBA au Canada, il convient de suivre étroitement les Lignes directrices KBA mondiales, en plus des lignes directrices présentées dans ce document et disponibles auprès du Secrétariat KBA canadien. Les Lignes directrices KBA mondiales comprennent des conseils pour proposer des KBA qui s'appuient sur des KBA existantes (par exemple, des ZICO qui peuvent être élargies pour inclure des taxons autres que les oiseaux), des KBA qui chevauchent étroitement les limites de gestion existantes (par exemple, les limites des aires protégées) et des KBA dont les nouvelles limites doivent être établies. Nous résumons ci-dessous les principaux éléments à prendre en compte pour délimiter les sites selon les Lignes directrices KBA mondiales, puis nous présentons des directives supplémentaires élaborées à partir de l'expérience acquise en matière d'identification et de délimitation des sites au Canada. Toute directive supplémentaire recherchée figurera probablement dans les Lignes directrices KBA mondiales. Il faut souligner que l'expérience en matière de délimitation des sites pour les espèces est beaucoup plus vaste; des directives sur la délimitation des KBA identifiées selon les critères écosystémiques seront élaborées en fonction de l'expérience acquise relativement à ces critères.

En plus de consulter des experts, les auteurs de propositions de KBA doivent consulter les détenteurs de droits et les parties prenantes, qui détiennent des informations pertinentes pour la délimitation pratique d'un site, en plus des informations sur les éléments de biodiversité du site. Chaque cas sera unique, et nous recommandons de suivre les lignes directrices mondiales et canadiennes, puis de faire preuve de jugement pratique et expert pour déterminer l'approche de délimitation la plus simple et la plus pertinente sur le plan écologique. Il est important de connaître le contexte pour appliquer les normes et les lignes directrices et, en fin de compte, pour prendre des décisions concernant les limites des KBA..

\section{Résumé des directives sur la délimitation tirées des Lignes directrices KBA mondiales}

Certains points saillants des Lignes directrices KBA mondiales sont repris ci-dessous par points.

\section{Concepts généraux}

- Il n'y a aucune exigence minimale ou maximale de taille pour les KBA. Les aspects de gestion doivent être pris en compte dans tous les cas, car un site doit être une unité gérable. Dans le cas des KBA, le potentiel de gestion désigne la possibilité d'un certain type de gestion efficace sur l'ensemble du site. Il s'agit d'une ligne directrice générale, qui se prête à un certain degré d'interprétation.

- Les limites des KBA mondiales ne doivent pas chevaucher celles des autres KBA mondiales. Les auteurs de propositions de KBA doivent s'efforcer d'harmoniser les limites proposées de la KBA avec celles qui existent déjà. Les directives concernant les KBA nationales et mondiales dont les limites se chevauchent sont différentes; voir les sections ci-dessous.

- Les jeux de données spatiales peuvent être examinés afin de déterminer quelles données peuvent être utiles pour la délimitation (par exemple, données topographiques, limites des types d'écosystèmes, couverture terrestre). 


\section{Détermination des limites initiales}

- Les cartes de répartition des espèces constituent un bon point de départ pour délimiter les endroits où il n'y a pas de sites existants dans la zone d'intérêt.

- Pour obtenir des éléments de biodiversité bien échantillonnés qualifiant une KBA, il est possible d'établir des cartes de répartition qui représentent leur étendue géographique locale connue à partir des données observées dans les localités. Pour les éléments comportant relativement peu de localités d'échantillonnage, il peut être nécessaire d'estimer l'étendue géographique approximative en se servant des connaissances sur les besoins en matière d'habitat, des cartes d'habitat et des modèles.

- Dans le cas des éléments de biodiversité qui n'occupent pas une KBA entière (par exemple, s'il y a lieu d'identifier une zone protégée existante comme une KBA), des cartes montrant leur répartition dans la KBA doivent être soumises avec la proposition de KBA. Des informations précises sur l'emplacement des éléments qualifiants permettront d'orienter leur gestion efficace. Cette couche de données spatiales supplémentaires sur les localités des éléments qualifiants est également importante pour évaluer les KBA en utilisant l'aire de répartition ou la zone d'occupation (ou tout autre paramètre de la zone), car elle permet de prouver que l'espèce se trouve dans le site.

- Les limites initiales des KBA peuvent être déterminées en tenant compte de la répartition des éléments de biodiversité qualifiants qui se chevauchent. Ces limites initiales des KBA doivent être déterminées de manière à ce que la zone qu'elles contiennent soit distincte des zones environnantes sur le plan de l'importance des éléments de biodiversité qualifiants ou de l'habitat, tout en réduisant au minimum l'inclusion de superficies de terres ou d'eau qui ne sont pas pertinentes pour les éléments de biodiversité qualifiants.

- Outre l'habitat, il est conseillé de prendre en compte les aspects spatiaux des limites écologiques, notamment la taille, la limite et la connectivité avec d'autres zones naturelles. En particulier, la détermination de limites qui s'harmonisent avec les caractéristiques naturelles de la topographie ou de l'habitat peut améliorer les perspectives de persistance des éléments de biodiversité qualifiants.

- Les populations d'espèces qualifiantes peuvent faire partie d'une métapopulation plus vaste; elles n'ont pas besoin d'être autosuffisantes. Elles doivent respecter les seuils fixés pour les unités reproductrices (UR).

- La délimitation des frontières écologiques dans les zones sauvages constituera un défi. Si des données précises sur les populations ne sont pas disponibles, des modèles de répartition des espèces peuvent être utilisés et validés par le biais d'enquêtes. Les données topographiques et environnementales telles que l'altitude, les lignes de crête, les caractéristiques géologiques, etc. peuvent être utilisées pour déterminer les limites.

\section{Aspects pratiques de la délimitation au Canada}

Il est important de retenir qu'il n'y a pas qu'une seule solution pour délimiter une KBA. De nombreuses décisions doivent être prises concernant la délimitation, compte tenu des lignes directrices, des jeux de données existants, de l'écologie des espèces/écosystèmes qualifiants et de l'avis des experts qui connaissent la zone où est situé le site. Tant que les limites du site lui permettent de répondre aux critères d'une KBA (en respectant les seuils quantitatifs) et que l'auteur de la proposition est convaincu que les directives les plus pertinentes et les avis des experts ont été suivis, les limites du site sont probablement satisfaisantes. 
Dans les cas où les auteurs de propositions sont incertains quant aux décisions de délimitation, ils sont invités à discuter des approches avec le Secrétariat KBA canadien et la Coalition KBA canadienne. Les limites des KBA doivent être, dans l'ordre suivant :

A. Significatives sur le plan écologique : Lors des ateliers et en consultation avec les experts des espèces et des écosystèmes, les questions de gestion doivent être mises de côté et la première étape de la délimitation doit être axée sur l'élaboration d'options de délimitation pertinentes sur le plan écologique.

B. Pertinentes pour la gestion ${ }^{11}$ : Les auteurs de propositions de KBA doivent tenir compte de l'emplacement des KBA existantes (par exemple, les ZICO) et des zones de conservation, de la propriété foncière et d'autres aspects de la gestion après l'établissement des limites écologiques initiales. Les aspects de gestion peuvent aider à choisir parmi plusieurs solutions de délimitation qui sont chacune rationnelles sur le plan écologique. Dans certains cas, les parties prenantes peuvent suggérer que les limites écologiques sont pertinentes pour la gestion et ne nécessitent pas d'autres modifications.

Il faut souligner que l'établissement de limites écologiquement significatives (décrites ci-dessous dans les étapes 1 et 2) doit faire appel à des experts en taxonomie, des experts en écosystèmes et d'autres personnes qui peuvent apporter des informations et une compréhension du paysage dans lequel la KBA sera située. Les propriétaires fonciers et autres détenteurs de droits et intervenants avisés peuvent également fournir des informations précises sur l'emplacement de l'habitat à l'intérieur des parcelles cadastrales, ou signaler des zones qui ne sont pas pertinentes pour les éléments de biodiversité en question, mais qui n'auront pas d'influence sur les limites de la KBA qui modifie l'importance de la biodiversité du site. Les étapes 3 et 4 peuvent être réalisées dans le cadre d'un processus distinct où d'autres types de contributions d'experts peuvent être plus pertinents, par exemple les experts en délimitation des KBA et les gestionnaires fonciers. Toutes les décisions relatives aux délimitations doivent être documentées et justifiées.

\section{Étape 1 : Définir des limites pertinentes sur le plan écologique pour les éléments qualifiants individuels}

L'objectif de la délimitation sera de prendre en compte une proportion suffisante de la taille de la population de l'espèce qualifiante ou de l'étendue de l'écosystème pour ensuite créer des limites qui ont une importance sur le plan écologique et de gestion. Les nouvelles KBA doivent d'abord être délimitées en fonction des aspects écologiques. Les instructions suivantes pour déterminer des limites de KBA écologiquement pertinentes sont également utiles pour tracer des limites autour d'éléments qualifiants individuels lorsque plusieurs éléments peuvent être inclus dans les limites de la KBA. La délimitation d'éléments qualifiants individuels qui ne sont pas les mêmes que les limites définitives des KBA se fait en combinant plusieurs éléments qualifiants, en ajoutant des éléments qualifiants à des KBA existantes, ou lorsque les limites des KBA sont délimitées sur la base de limites de gestion existantes (par exemple, lorsque la limite d'une zone protégée existante a une longueur et une forme appropriées pour servir de limite à une KBA). Le site ne doit pas contenir une population autonome de l'espèce qualifiante, mais doit répondre à des exigences seuils. Par ailleurs, il n'existe pas de prescription précise pour délimiter un site. Voici une série de mesures

11 À noter que l'idée même de «site» suppose une facilité de gestion. Les KBA doivent être délimitées selon une logique écologique, mais elles doivent aussi, de préférence, être pratiques à gérer. Par exemple, si l'emplacement des éléments de biodiversité qualifiants se situe dans une zone de conservation ou d'intendance existante, ces limites peuvent être appropriées pour la KBA. Lors des premiers projets de KBA au Canada, les gestionnaires et les planificateurs des terres ont indiqué que les sites délimités de manière écologique sont préférables. 
à prendre pour délimiter un nouveau site. Nous fournissons quelques suggestions de couches de données qui sont propres aux écosystèmes terrestres et aquatiques; cette information sera étoffée et disponible sur le site Web des KBA. Il est à signaler que l'expérience en matière de délimitation des sites marins et côtiers est limitée et que des conseils en matière de délimitation seront ajoutés ultérieurement pour ces systèmes.

\section{Systèmes terrestres}

Les systèmes terrestres ne comportent pas toujours de limites écologiques évidentes, contrairement à certains systèmes d'eau douce, par exemple lorsque les limites des lacs et des rivières sont claires. Les approches suivantes peuvent être adoptées :

En ce qui concerne les espèces qualifiantes, vous devrez délimiter les occurrences d'élément (OE) pertinents ou d'autres observations en utilisant des données spatiales pertinentes. Les occurrences d'éléments et les caractéristiques d'origine (NatureServe, 2020) constituent de bons éléments de base pour délimiter des KBA au Canada. Les couches de données spatiales suivantes peuvent être utilisées pour délimiter les endroits l'espèce a été observée :

- Données topographiques, si la répartition de l'élément est liée à l'altitude;

- Géologie superficielle/du substratum rocheux, climat, sol, agents de perturbation naturels;

- Informations sur les habitats des espèces, y compris les couches de données sur les habitats essentiels, les catégories de couverture terrestre pertinentes, les modèles d'espèces, les photographies aériennes, les images satellites, etc.;

- Informations sur les bassins versants si la gestion des espèces est plus pertinente à cette échelle;

- Données sur la couverture terrestre non liées à l'habitat, s'il existe des informations sur les infrastructures bâties, les routes, etc. qui divisent le paysage et créent des zones contiguës qui répondent aux critères d'une KBA;

- S'il n'y a pas de couches de données spatiales pertinentes pour orienter la délimitation, vous pouvez également effectuer une délimitation stricte autour des occurrences d'élément (OE) ou des observations, en respectant une zone tampon minimale si une observation comporte une incertitude spatiale.

- Si des paramètres fondés sur la superficie sont utilisés pour vérifier l'atteinte des seuils liés à un critère d'espèce, il faut inclure dans la limite de la KBA une superficie minimale de la zone d'occupation/ de l'étendue de l'habitat approprié/de l'aire de répartition à l'échelle mondiale ou nationale. Nous recommandons de commencer par les données d'observation et de déterminer quel est l'habitat le plus pertinent qui doit, en fonction de ces observations, être pris en compte à l'intérieur des limites finales.

- En ce qui concerne les écosystèmes qualifiants, il faut utiliser des cartes de l'étendue des écosystèmes, des modèles prédictifs d'écosystèmes, des données sur la couverture terrestre, des photos aériennes ou des images satellites pour cartographier l'étendue de l'écosystème et déterminer la superficie nécessaire pour atteindre les seuils. Comme l'expérience à ce jour en matière de cartographie des KBA axées sur les écosystèmes demeure limitée, nous prévoyons de mettre à jour ce document et d'ajouter des directives à ce sujet en fonction de l'expérience acquise.

- Les experts doivent être consultés pour déterminer la superficie exacte qui doit être incluse dans la KBA. 
Il faut savoir qu'il y aura probablement des zones au sein de la KBA qui ne sont pas pertinentes pour les espèces ou les écosystèmes qualifiants, et la superficie à inclure est laissée à la discrétion de l'auteur de la proposition, lequel doit prendre conseil auprès d'experts connaissant la zone. De manière générale, il convient d'inclure le moins de terres possible qui ne sont pas pertinentes pour l'espèce ou l'écosystème, mais surtout dans le cas des grandes KBA, il est inévitable d'inclure des terres non pertinentes. La justification de la délimitation de la KBA doit être bien documentée et sera examinée par des experts des espèces/écosystèmes ainsi que par des experts de la Norme et des Lignes directrices KBA.

\section{$\underline{\text { Systèmes aquatiques }}$}

Pour l'essentiel, les KBA dans les systèmes aquatiques seront identifiées pour les espèces en péril et les espèces géographiquement restreintes (critères A1 et B1).

- Les experts canadiens en matière d'eau douce ont indiqué qu'une délimitation stricte autour des éléments qualifiants (comme pour les espèces et les écosystèmes terrestres) constitue l'approche la plus appropriée pour délimiter les KBA d'eau douce - et plus précisément pour les éléments qui sont entièrement aquatiques. Ces directives se concrétisent par la cartographie de sections précises de cours d'eau et de rivières, ainsi que de parties de lacs ou de lacs entiers. Une zone tampon minimale de 30 mètres est suggérée pour délimiter les lacs et les rivières afin de tenir compte des imprécisions des données spatiales et des variations interannuelles.

- Il a été question de l'utilisation de zones tampons plus grandes pour prendre en compte l'interaction entre les écosystèmes terrestres et aquatiques, et l'unité responsable de la biodiversité d'eau douce de l'UICN a recommandé d'utiliser les données du sous-bassin hydrographique HydroBASINS de niveau 12 (Lehner and Grill, 2013) pour délimiter les KBA d'eau douce. Il s'agit d'une autre possibilité; c'est pourquoi nous recommandons de demander l'avis d'experts sur la délimitation de sites en particulier afin de s'assurer que la délimitation des KBA tient compte des éléments les plus pertinents. Il peut s'agir d'une bonne solution de délimitation s'il existe à proximité des espèces terrestres qualifiantes.

- Lorsque des éléments qualifiants aquatiques et des éléments qualifiants à proximité sont présents dans les milieux terrestres environnants, il faut faire appel au jugement d'experts pour déterminer si ces éléments doivent être intégrés dans une seule KBA et pour déterminer l'approche la plus pertinente de délimitation de la KBA.

- Dans certaines régions du Canada, on a également mis à l'essai la possibilité de délimiter le pourtour des lacs et des zones humides à l'aide de courbes de niveau qui délimitent plus largement ces habitats. Les experts doivent examiner la courbe de niveau spécifique et la nécessité de la couper manuellement lorsqu'elle s'étend trop loin. Le fait que les espèces qualifiantes vivent dans des milieux aquatiques ou humides influera sur la méthode de délimitation de la KBA.

- En ce qui concerne les lacs, la délimitation du lac lui-même peut être effectuée à l'aide de la Global Lakes and Wetlands Database (Lehner et Doell, 2004) ou d'autres jeux de données locaux ou régionaux similaires (et probablement plus précis) qui incluent les lacs. 


\section{Étape 2 : Intégrer plusieurs éléments qualifiants dans une seule KBA}

Si plusieurs éléments qualifiants sont présents dans une zone, la première étape consiste à évaluer quelles zones précises répondraient aux critères d'une KBA pour chaque élément qualifiant (en respectant les seuils quantitatifs). Si plusieurs zones qui se chevauchent ou se rapprochent les unes des autres répondent aux critères de différents éléments qualifiants, les limites de la KBA doivent être tracées strictement autour du groupe d'éléments de biodiversité qualifiants, soit en utilisant des jeux de données spatiales pertinents (par exemple, des lignes d'élévation), soit en traçant une limite à la main (par exemple, une délimitation stricte autour des éléments de biodiversité, avec une zone tampon minimale de $30 \mathrm{~m}$, le cas échéant). Si les éléments de biodiversité admissibles sont éloignés les uns des autres, mais sont tous inclus dans une limite de gestion existante (par exemple un parc national), cette limite pourrait être utilisée comme limite de la KBA, selon que la taille de la limite de gestion est comparable au groupe d'éléments qualifiants de la KBA. S'il n'y a pas de limite de gestion existante ou proposée, la décision de délimiter les éléments séparément ou ensemble doit reposer sur la distance entre les éléments (bien qu'il n'y ait pas de distance seuil), sur la compréhension des besoins écologiques des différents éléments qualifiants ainsi que sur la connaissance du paysage et des milieux. Il n'y a pas une seule bonne façon de prendre la décision. Si les différents éléments qualifiants ne partagent aucune exigence en matière d'habitat, ils pourraient être délimités séparément. S'il y a des chevauchements entre les exigences relatives aux habitats, ils pourraient être délimités ensemble. Dans certains cas, les éléments qualifiants peuvent être répartis sur plusieurs sites, dans la mesure où chacun d'entre eux respecte les seuils des critères et où il n'y a pas de chevauchement spatial entre les KBA. De manière générale, les KBA doivent être des polygones simples sans trous (il peut y avoir des exceptions à cette règle pour les KBA plus grandes). En cas de doute, consultez le Secrétariat et la Coalition KBA pour obtenir des précisions.

\section{Étape 3 : Intégrer des éléments qualifiants dans des KBA existantes}

L'identification et la délimitation des KBA en fonction de plusieurs critères et éléments qualifiants posent un problème de séquence - il est possible que des éléments supplémentaires doivent être ajoutés aux KBA existantes qui ont été délimitées à différents moments. Il n'y a pas de solution facile à ce problème, et nous prévoyons que les limites des KBA devront être ajustées périodiquement à mesure que d'autres KBA seront identifiées. Cette situation est actuellement la plus pertinente en ce qui concerne les ZICO existantes qui ont été désignées au cours des dernières décennies au Canada. Les directives suivantes s'appliquent à ces situations :

- Si les limites écologiques d'un ou de plusieurs éléments qualifiants se situent entièrement ou en grande partie dans les limites d'une KBA existante, la limite de la KBA existante doit être utilisée pour la délimitation. Les données relatives aux nouveaux éléments de biodiversité qualifiants doivent être ajoutées aux données d'admissibilité de la KBA existante.

- Si la nouvelle KBA proposée s'étend au-delà des limites d'une KBA existante, la ou les zones supplémentaires doivent :

a. Être exclues si elles ne sont pas nécessaires pour atteindre le seuil du critère pertinent et si elles ne sont pas pertinentes sur le plan écologique.

b. Être ajoutées à la limite de la KBA existante. Dans ce cas, une proposition de modification doit être envoyée avec justification à l'auteur de la proposition de la KBA originale par l'intermédiaire de la Coalition KBA canadienne. 
c. Être délimitées comme une KBA distincte et adjacente. Cette situation est possible si les limites initiales ne peuvent être modifiées pour quelque raison que ce soit (par exemple, un processus politique est associé à ces limites). La nouvelle KBA adjacente doit répondre de manière indépendante aux critères d'une KBA.

d. Faire l'objet d'une consultation auprès de la Coalition KBA si la zone initiale et la nouvelle zone sont nécessaires pour qu'un site soit admissible et que les limites initiales ne peuvent être modifiées.

- Si la nouvelle KBA proposée englobe entièrement une KBA existante, il est possible de proposer de nouvelles limites pour une KBA qui intègre tous les éléments qualifiants (en consultation avec les auteurs de la proposition de KBA initiale).

- Si les limites initiales d'une KBA ne sont pas pertinentes du point de vue écologique ou de la gestion (par exemple, les KBA plus anciennes peuvent avoir été délimitées selon un ensemble différent de lignes directrices et moins informatives), des consultations avec les auteurs des propositions de sites initiaux sont nécessaires pour ajuster les limites initiales afin de prendre une décision quant aux prochaines étapes.

- Dans toutes les possibilités énumérées ci-dessus, s'il existe plusieurs éléments qualifiants de KBA, la zone pertinente pour chaque élément qualifiant de KBA doit être cartographiée, en plus des limites définitives de la KBA qui comprend tous les éléments qualifiants. Des informations sur la manière de cartographier les éléments qualifiants de KBA figurent à l'étape 1.

\section{Étape 4 : Prendre en compte les aspects de gestion, y compris les limites de gestion existantes, dans la délimitation}

- Si l'échelle des limites de gestion existantes est pertinente à la délimitation d'une nouvelle KBA, les limites existantes dans lesquelles une gestion active est en cours doivent être utilisées.

- Si les limites de gestion existantes ne conviennent pas aux éléments qualifiants de la KBA, il est possible de proposer une KBA qui chevauche une zone de conservation ou qui est située à l'intérieur de celle-ci.

- Dans les cas où la zone de conservation existante est beaucoup plus étendue que la zone d'intérêt comprenant les éléments qualifiants de la KBA, les limites de la KBA doivent être établies à une échelle qui éclaire le plus possible les décisions de gestion, plutôt que d'adopter les limites de la zone de conservation comme limites de la KBA.

- Un site peut comprendre plusieurs limites pertinentes existantes dans les environs (par exemple une KBA préexistante, ainsi qu'une ou plusieurs zones de conservation). Dans des cas comme celui-ci, il est difficile de déterminer quelle limite il faut utiliser (le cas échéant) et comment intégrer les éléments qualifiants de la KBA dans la combinaison.

- Les limites existantes de la KBA doivent être retenues, si elles sont raisonnables.

- Si les limites actuelles de la KBA ne conviennent pas à des espèces qualifiantes additionnelles, il faut en discuter avec les auteurs de la proposition de la KBA initiale afin de les modifier. 
- Si les limites de la zone de conservation sont plus pertinentes d'un point de vue écologique que les limites actuelles de la KBA, il faut en discuter avec les auteurs de la proposition de KBA initiale.

- Dans certains cas où les limites de la KBA ont été établies il y a longtemps et sans avoir un niveau de précision élevé, il peut être utile de collaborer avec les auteurs de la proposition initiale pour suggérer de nouvelles limites.

- Dans certains cas, les éléments qualifiants de la KBA peuvent être divisés en plusieurs sites, chacun d'entre eux respectant toujours les seuils des critères. Dans ces cas, les solutions de délimitation peuvent varier, allant d'une grande KBA à une douzaine de petites KBA, qui pourraient toutes respecter les seuils des critères. Il convient de discuter avec les experts pour déterminer si une ou plusieurs KBA sont plus appropriées en fonction, notamment, de la distance qui sépare les sites potentiels, de l'écologie et du comportement de l'espèce qualifiante ainsi que de la couverture terrestre entre les sites potentiels. En outre, il peut également être utile de réfléchir à la manière dont elles peuvent être intégrées aux multiples limites de gestion existantes.

- Il faut communiquer avec les gestionnaires fonciers et les détenteurs de droits dans la zone pour leur demander de fournir des renseignements sur le site qui pourraient être utiles à la délimitation. Les limites peuvent être modifiées afin de tenir compte des aspects de gestion si le changement est justifié et si les critères de la KBA sont toujours respectés dans les limites.

- Dans toutes les possibilités énumérées ci-dessus, s'il existe plusieurs éléments qualifiants de KBA, la zone pertinente pour chaque élément qualifiant de KBA doit être cartographiée, en plus de la limite définitive de la KBA qui comprend tous les éléments qualifiants. Des informations sur la manière de cartographier les éléments qualifiants de KBA figurent à l'étape 1.

\section{Intégration des sites KBA mondiaux et nationaux}

Une question qui se posera régulièrement au Canada concerne la nécessité d'intégrer les informations sur les espèces qualifiantes mondiales et nationales dans les KBA qui se chevauchent. Notre expérience à ce jour montre que des situations complexes se produisent fréquemment lorsque plusieurs KBA mondiales et nationales sont très proches les unes des autres et que, si elles étaient délimitées séparément, elles se chevaucheraient. Les KBA mondiales ne peuvent pas chevaucher d'autres KBA mondiales, pour plusieurs raisons, notamment la nécessité de proposer un «site» distinct qui puisse être géré ou supervisé de manière appropriée. Tout chevauchement de ce type doit être résolu en combinant les KBA en une seule KBA plus importante ou en créant des KBA adjacentes. Dans la mesure du possible, c'est également la recommandation à suivre pour délimiter les KBA nationales. Toutefois, dans certaines circonstances où il n'est pas possible de regrouper une KBA mondiale et une KBA nationale ou deux KBA nationales, des chevauchements peuvent être autorisés. Les auteurs de propositions doivent d'abord communiquer avec le Secrétariat KBA pour obtenir des conseils le cas échéant.

Les directives suivantes sur l'intégration des KBA mondiales et nationales sont fondées sur les premières expériences de délimitation des sites aux deux niveaux, et il est probable que des principes supplémentaires seront établis en fonction de l'expérience acquise.

- Un site peut répondre aux critères d'une KBA mondiale et d'une KBA nationale pour des éléments de biodiversité additionnels. Si les limites sont les mêmes pour chaque niveau de KBA, un formulaire de proposition mondial doit être rempli, et la liste additionnelle des espèces qualifiantes nationales doit être incluse à titre de «biodiversité supplémentaire». Dans la mesure du possible, les éléments qualifiants nationaux doivent être évalués pour ces sites, et les éléments qualifiants nationaux qui respectent les seuils sur le site doivent être pris en note. 
- Si un site répond à la fois aux critères d'une KBA mondiale et d'une KBA nationale pour des éléments de biodiversité supplémentaires, et que les limites de chaque niveau sont différentes, la localisation des éléments qualifiants nationaux peut avoir une incidence sur les limites de la KBA mondiale. Si un élément qualifiant national est présent en quantité suffisante pour respecter les seuils de la KBA, cet aspect de gestion doit avoir une incidence sur la délimitation de la KBA mondiale. Les limites de la KBA doivent être élargies afin de tenir compte des deux niveaux d'information sur les éléments qualifiants. La nécessité d'intégrer plusieurs éléments qualifiants peut dépendre de l'emplacement précis des différents éléments, et il faut consulter les experts pour savoir si la meilleure solution consiste à regrouper ces éléments sur un seul site ou à les séparer.

- Un site peut avoir déjà répondu aux critères d'une KBA nationale et répondre désormais aux critères d'une KBA mondiale pour d'autres éléments qualifiants ou inversement. La Coalition KBA canadienne doit être consultée avant toute décision concernant la délimitation finale.

- S'il n'est pas nécessaire de modifier les limites, il n’y a pas de problème et les limites existantes doivent être retenues.

- Si les limites doivent être élargies, il sera alors important de discuter avec les auteurs de la proposition du site initial et de négocier de nouvelles limites qui demeurent pertinentes pour les groupes responsables de la gestion de la zone. De manière générale, même si le site est initialement une KBA nationale, son existence et toute sa gestion actuelle nécessitent de prendre en considération les frontières nationales et de les respecter dans la mesure du possible.

- Dans tous les cas, les emplacements précis des éléments de biodiversité qualifiants doivent être indiqués dans une couche spatiale distincte de la couche spatiale des limites externes de la KBA.

\section{Comment élaborer des fichiers de formes KBA afin de soumettre les limites des KBA}

Les directives concernant la préparation des couches de données spatiales sur les limites des KBA sont présentées dans l'annexe B du processus de proposition de KBA mondiale (Secrétariat KBA, 2019). Deux types de couches spatiales pour la soumission des KBA remplissent des fonctions différentes : 1) les limites de la KBA, et 2) les emplacements précis des éléments de biodiversité qualifiants à l'intérieur des limites de la KBA. Il s'agit de données importantes pour éclairer la gestion judicieuse du paysage. De vastes zones peuvent être incluses dans les KBA pour diverses raisons, et la couche de données sur les limites des KBA peut donc être moins utile pour déterminer les zones où certaines activités doivent être interdites (par exemple, la randonnée dans un parc national). Dans certains cas, les deux couches peuvent être identiques, ce qui doit être précisé. Lorsque les limites définitives de la KBA sont retenues à partir de zones de conservation ou d'intendance existantes, il est préférable de soumettre également la deuxième catégorie de fichier de données spatiales afin de disposer d'informations précises sur les éléments de biodiversité qui pourraient devoir être gérés en particulier. Étant donné que les données de localisation précises ne sont pas toujours disponibles pour certaines espèces ou qu'elles peuvent être sensibles, cette deuxième catégorie de fichier de données spatiales n'est pas nécessaire, mais elle doit être fournie dans la mesure du possible. Toutes les limites des KBA doivent être vérifiées auprès des autorités provinciales et territoriales responsables des espèces en péril (d'abord par l'intermédiaire du Secrétariat KBA canadien, qui dresse une liste des espèces sensibles et des restrictions en matière de données par province/territoire) afin de s'assurer que les données sur les espèces sensibles sont gérées de manière appropriée et que les limites des KBA pour ces espèces sont établies conformément aux politiques provinciales ou territoriales. 


\section{RÉFÉRENCES}

Baldwin, K., K. Chapman, D. Meidiner, P. Uhlig, L. Allen, S. Basquill, D. Faber-Langendoen, N. Flynn, C. Kennedy, W. Mackenzie, M. Major, W. Meades, C. Morneau et J.-P. Saucier. 2019. Classification nationale de la végétation du Canada : principes, méthodes et état. Ressources naturelles Canada, Service canadien des forêts. Rapport d'information GLC-X-23: 162 p.

Beresford, A. E., G. M. Buchanan, P. F. Donald, S. H. M. Butchart, L. D. C. Fishpool et C. Rondinini. 2011. Minding the protection gap: estimates of species' range sizes and holes in the Protected Area network. Animal Conservation 14:114-116.

CCCEP (Conseil canadien pour la conservation des espèces en péril). 2016. Espèces sauvages 2015 : La situation générale des espèces au Canada. Groupe de travail national sur la situation générale : $128 \mathrm{p}$. Accessible à l'adresse https://wildlife-species.canada.ca/species-risk-registry/virtual_sara/files/reports/Especes\%20sauvages\%20 2015.pdf.

Chu, C., C. K. Minns, N. P. Lester et N. E. Mandrak. 2015. An updated assessment of human activities, the environment, and freshwater fish biodiversity in Canada. Canadian Journal of Fisheries and Aquatic Sciences 72(1):135-148. Accessible à l'adresse http://dx.doi.org/10.1139/cjfas-2013-0609.

COSEPAC (Comité sur la situation des espèces en péril au Canada). 2018. Lignes directrices du COSEPAC pour reconnaître les unités désignables. Accessible à l'adresse https://cosewic.ca/index.php/fr/rapports/preparationrapports-situation/lignes-directrices-reconnaitre-unites-designables.

COSEPAC (Comité sur la situation des espèces en péril au Canada). 2019. Définitions et abréviations du COSEPAC. $<$ https://cosewic.ca/index.php/fr/a-propos-de-nous/definitions-abreviations $>$. Téléchargé le 13 novembre 2020.

COSEPAC (Comité sur la situation des espèces en péril au Canada). 2020a. Bref historique : Comité sur la situation des espèces en péril au Canada. <https://cosewic.ca/index.php/fr/a-propos-de-nous/bref-historique>. Téléchargé le 5 octobre 2020 .

COSEPAC (Comité sur la situation des espèces en péril au Canada). 2020b. Espèces sauvages canadiennes en péril 2020. <https://www.canada.ca/fr/environnement-changement-climatique/services/registre-public-especes-peril/ publications/especes-sauvages-canadiennes-peril-2020.html>. Téléchargé le 13 novembre 2020.

ECCC (Environnement et Changement climatique Canada) 2019. Rapport sommaire du sixième rapport national du Canada à la Convention sur la diversité biologique. Gouvernement du Canada, Gatineau (Québec) Canada. Accessible à l'adresse https://biodivcanada.chm-cbd.net/sites/biodivcanada/files/inline-files/FR_Rapport\%20Sommaire\%20 Canada\%20a\%201a\%20CDB_Final_2.pdf.

Eisenberg, J. F. 1977. The evolution of the reproductive unit in the Class Mammalia. In Rosenblatt, J. S., and B. R. Komisaruk, editors. Reproductive Behavior and Evolution. Plenum Publishing Corporation, New York City, New York, USA.

Faber-Langendoen, D., J. Nichols, L. Master, K. Snow, A. Tomaino, R. Bittman, G. Hammerson, B. Heidel, L. Ramsay, A. Teucher et B. Young. 2012. NatureServe Conservation Status Assessments: Methodology for Assigning Ranks. NatureServe, Arlington, VA, USA.

Faber-Langendoen, D., K. Baldwin, R. K. Peet, D. Meidinger, E. Muldavin, T. Keeler-Wolf et C. Josse. 2018. The EcoVeg approach in the Americas: US, Canadian and international vegetation classifications. Phytocoenologia 48(2):215-237.

Faber-Langendoen, D., T. Keeler-Wolf, D. Meidinger, D. Tart, B. Hoagland et al. 2014. EcoVeg: a new approach to vegetation description and classification. Ecological Monographs 84:533-561.

Fraser, D. 2019. Discussion Paper : Adapting the Global Key Biodiversity Standard for National Use in Canada. Rapport non publié destiné à Environnement et Changement climatique Canada. 10 décembre 2019.

Gärdenfors, U., C. Hilton-Taylor, G. M. Mace et J. P. Rodríguez. 2001. The application of UICN Red List Criteria at regional levels. Conservation Biology 15:1206-1212. 
GFPTC (gouvernements fédéral, provinciaux et territoriaux du Canada). 2010. Biodiversité canadienne, état et tendances des écosystèmes en 2010. Conseils canadiens des ministres des ressources, Ottawa, Ontario, Canada. Accessible à https://biodivcanada.chm-cbd.net/sites/biodivcanada/files/2018-01/FR_Biodiversit\%C3\%A9_ canadienne_COMPLET_0.pdf.

Gouvernement du Canada. 2020. Le Canada se joint à la coalition de la haute ambition pour la nature et les peuples. Communiqué de presse d'Environnement et Changement climatique Canada. 28 septembre 2020. Ottawa, Ontario. Canada.

ICOAN-Canada (Initiative de conservation des oiseaux d'Amérique du Nord). 2019. L'état des populations d'oiseaux du Canada. Environnement et Changement climatique Canada, Ottawa, Canada. Accessible à http://nabci.net/ ressources/letat-des-populations-doiseaux-du-canada-2019/?lang=fr.

IUCN (International Union for the Conservation of Nature) Standards and Petitions Subcommittee. 2014. Guidelines for Using the IUCN Red List Categories and Criteria. Version 11. Prepared by the Standards and Petitions Subcommittee. Available at http://www.iucnredlist.org/documents/RedListGuidelines.pdf.

KBA (Key Biodiversity Areas) Secretariat. 2019. Key Biodiversity Areas Proposal Process: Guidance on Proposing, Reviewing, Nominating and Confirming sites. Version 1.0. Prepared by the KBA Secretariat and KBA Committee of the KBA Partnership. Cambridge, UK. Available at http://www.keybiodiversityareas.org/working-with-kbas/ proposing-updating/proposal-process.

KBA (Key Biodiversity Areas) Standards and Appeals Committee. 2020. Guidelines for using A Global Standard for the Identification of Key Biodiversity Areas. Version 1.1. Prepared by the KBA Standards and Appeals Committee of the IUCN Species Survival Commission and IUCN World Commission on Protected Areas. IUCN, Gland, Switzerland. https://portals.iucn.org/library/node/49131.

Keith, D. A., J. P. Rodríguez, K. M. Rodríguez-Clark, E. Nicholson, K. Aapala, A. Alonso, M. Asmussen, S. Bachman, A. Basset, E. G. Barrow et J. S. Benson. 2013. Scientific foundations for an UICN Red List of Ecosystems. PLOS one 8(5):e62111.

Kraus, D. et A. Hebb. 2020. Southern Canada's crisis ecoregions: identifying the most significant and threatened places for biodiversity conservation. Biodiversity and Conservation:1-18. Accessible à https://doi.org/10.1007/ s10531-020-02038-x.

Lehner, B. et G. Grill. 2013. Global river hydrography and network routing: baseline data and new approaches to study the world's large river systems. Hydrological Processes 27(15):2171-2186. Données accessibles à www. hydrosheds.org.

Lehner, B. et P. Doell. 2004. Development and validation of a global database of lakes, reservoirs and wetlands. Journal of Hydrology 296/1-4:1-22. Données accessibles à https://www.worldwildlife.org/pages/global-lakes-andwetlands-database.

Mounce, R., M. Rivers, S. Sharrock, P. Smith et S. Brockington. 2018. Comparing and contrasting threat assessments of plant species at the global and sub-global level. Biodiversity and Conservation:907-930.

NatureServe. 2018. Biotics 5: Web-Enabled Biodiversity Information Management System. <http://www.natureserve. org/conservation-tools/biotics-5>. Téléchargé le 5 octobre 2020.

NatureServe. 2020. Conservation Tools and Services. <https://www.natureserve.org/conservation-tools/natureservelearning-center/fundamental-skill-building/data-use-training>. Téléchargé le 5 octobre 2020.

Rodríguez, J.P. 2008. National Red Lists: the largest global market for UICN Red List Categories and Criteria. Endangered Species Research 6:193-198.

UICN (Union Internationale pour la Conservation de la Nature). 2012. Catégories et critères de l'UICN pour la liste rouge : version 3.1. Deuxième édition. UICN, Gland, Suisse et Cambridge, Royaume-Uni.

UICN (Union Internationale pour la Conservation de la Nature). 2016. Standard mondial pour l'identification des Zones Clés pour la Biodiversité, version 1,0 Première édition UICN, Gland, Suisse. Accessible à https://portals.iucn. org/library/sites/library/files/documents/2016-048-Fr.pdf. 


\section{ANNEXE 1 PRÉCISIONS SUPPLÉMENTAIRES CONCERNANT LES CRITÈRES ET LES SEUILS DE LA NORME NATIONALE}

La présente section contient des précisions supplémentaires concernant l'application des critères de le Standard KBA national. Vous y trouverez des directives sur les cas peu courants qui peuvent se présenter lors de l'évaluation de la conformité des éléments de biodiversité aux critères KBA, ainsi que sur les conflits entre les différents systèmes d'évaluation.

\subsection{Précisions supplémentaires concernant l'application du critère A1}

\subsubsection{Identification des espèces qualifiantes selon le critère national A1}

La catégorie de la Liste rouge de l'UICN, le rang G, la catégorie de statut du COSEPAC et le rang N d'un taxon déterminent s'il est considéré comme menacé à l'échelle nationale, et à quel niveau, comme le montre le tableau suivant. Les espèces qualifiantes selon le critère national A1 sont incluses dans les rangées en jaune du tableau ci-dessous. Seuls les rangs G, les rangs N et les catégories de statut du COSEPAC attribués depuis moins de 12 ans sont pris en compte, et seules les catégories de l'UICN attribuées depuis moins de 10 ans sont prises en compte. Dans certains cas, l'utilisation d'un classement plus ancien est permise, si une justification convaincante est fournie (par exemple, les tendances de population sont restées stables et la réévaluation est simplement en retard); voir la section VII pour de plus amples informations à ce sujet.

\begin{tabular}{|c|c|c|c|c|}
\hline & \multicolumn{2}{|c|}{ Tous les taxons } & \multicolumn{2}{|c|}{ Taxons endémiques au Canada seulement } \\
\hline & $\begin{array}{l}\text { Catégorie de statut du } \\
\text { COSEPAC }\end{array}$ & Rang N & $\begin{array}{l}\text { Catégorie de la Liste } \\
\text { rouge de I'UICN }\end{array}$ & Rang G \\
\hline $\begin{array}{l}\text { Possiblement disparu } \\
\text { ou possiblement dis- } \\
\text { paru au Canada }{ }^{12}\end{array}$ & - & $\begin{array}{l}\text { Possiblement disparu } \\
\text { (NH) }\end{array}$ & $\begin{array}{l}\text { Possiblement disparu } \\
\text { (CR(PE)) ou Possiblement } \\
\text { disparu à l'état sauvage } \\
\text { (CR(PEW)) }\end{array}$ & $\begin{array}{l}\text { Possiblement disparu (GH ou } \\
\mathrm{TH} \text { ) ou Possiblement disparu } \\
\text { à l'état sauvage (GHC ou } \\
\mathrm{THC} \text { ) }\end{array}$ \\
\hline $\begin{array}{l}\text { Menacé à l'échelle } \\
\text { nationale au niveau } 1\end{array}$ & $\begin{array}{l}\text { En voie de disparition } \\
\text { (ND) }\end{array}$ & Gravement en péril (N1) & $\begin{array}{l}\text { En danger critique (CR) } \\
\text { ou en danger (EN) }\end{array}$ & $\begin{array}{l}\text { Gravement en péril (G1 ou } \\
\text { T1) }\end{array}$ \\
\hline $\begin{array}{l}\text { Menacé à } \\
\text { l'échelle nationale } \\
\text { au niveau } 2\end{array}$ & $\begin{array}{l}\text { Espèce menacée } \\
\text { (M) }\end{array}$ & En péril (N2) & Vulnérable (VU) & En péril (G2 ou T2) \\
\hline $\begin{array}{l}\text { Non menacé } \\
\text { à l'échelle } \\
\text { nationale }\end{array}$ & $\begin{array}{l}\text { Préoccupante } \\
(\mathrm{P}), \text { non en péril } \\
\text { (NEP) ou données } \\
\text { insuffisantes (DI) }\end{array}$ & $\begin{array}{l}\text { Vulnérable (N3), } \\
\text { apparemment en } \\
\text { sécurité (N4), en } \\
\text { sécurité (N5) ou } \\
\text { inclassable (NU) }\end{array}$ & $\begin{array}{l}\text { Quasi menacé } \\
\text { (NT), préoccupation } \\
\text { mineure (LC) } \\
\text { ou données } \\
\text { insuffisantes (DD) }\end{array}$ & $\begin{array}{l}\text { Vulnérable (G3 ou } \\
\text { T3), apparemment en } \\
\text { sécurité (G4 ou T4), en } \\
\text { sécurité (G5 ou T5) ou } \\
\text { inclassable (GU ou TU) }\end{array}$ \\
\hline
\end{tabular}

12 L'identification d'une KBA nationale pour un taxon qui est possiblement disparu ou possiblement disparu au Canada n'est autorisée que conformément au sous-critère A1e, dans les rares cas où il est justifié de croire que le taxon est toujours présent sur le site bien qu'il n'ait pas été repéré. 
Dans les cas où un taxon se voit attribuer des rangs appartenant à plus d'une rangée dans le tableau ci-dessus, collectivement appelés «rangs contradictoires», les règles suivantes s'appliquent :

1. Si les rangs conflictuels ont été attribués à plus de cinq ans d'intervalle, utilisez le rang le plus récent.

2. Si les rangs conflictuels ont été attribués à moins de cinq ans d'intervalle, utilisez le rang le plus prudent, à savoir celui qui correspond à la catégorie de risque la plus élevée (c'est-à-dire à la rangée la plus élevée du tableau), sauf s'il existe une bonne raison de ne pas le faire (par exemple, si de nouvelles informations ont conduit à une réévaluation dans un système plus adéquat et plus récent).

3. S'il existe une bonne raison de privilégier un système d'évaluation du statut par rapport à un autre pour une espèce ou un taxon, il faut fournir une justification complémentaire si les règles ci-dessus ne sont pas respectées.

Si le taxon est considéré comme menacé à l'échelle nationale, le rang le plus récent à l'origine de cette détermination est appelé «rang qualifiant».

\subsection{2 Éléments qualifiants potentiels du Standard KBA mondial qui répondent au critère A1 national}

Certains taxons classés par NatureServe comme menacés à l'échelle mondiale (rangs G de G1, G2, T1 ou T2) ne sont pas considérés comme des espèces qualifiantes selon le critère A1 du Standard KBA mondial, en raison de la prépondérance des évaluations de la Liste rouge de l'UICN dans le Standard KBA mondial et parce que les KBA mondiales ne peuvent pas être désignées pour les taxons infraspécifiques. Ces taxons appartiennent à au moins une des cinq catégories décrites ci-dessous.

1. Le taxon est classé G2G4, et est donc considéré comme G3 aux fins du Standard KBA mondial.

2. Le taxon fait partie d'une catégorie de la Liste rouge de l'UICN qui n'est plus à jour (c'est-à-dire qui a plus de 10 ans), et ne peut donc pas être un élément qualifiant selon le critère A1 mondial tant que sa catégorie de l'UICN n'aura pas été réévaluée.

3. Le taxon fait partie d'une catégorie de la Liste rouge de l'UICN qui le disqualifie du critère A1 du Standard KBA mondial (par exemple DD).

4. Le taxon est présent en dehors des États-Unis et du Canada, et son rang G ne peut donc pas être utilisé dans les évaluations selon le critère A1 mondial.

5. Le taxon est infraspécifique et ne peut donc pas entraîner une proposition de KBA mondiale.

Il existe au Canada des taxons appartenant à toutes ces catégories et qui ne sont pas autrement des éléments qualifiants selon le critère A1 national. Ces taxons seront acceptés comme éléments qualifiants selon le critère A1 de le Standard KBA national, auquel cas les règles de résolution des contradictions entre les rangs de menace nationaux (section 1.1.1) doivent être appliquées pour résoudre les contradictions entre les rangs de menace mondiaux. Lorsque des KBA nationales selon le critère A1 sont proposées pour des taxons menacés à l'échelle mondiale, les seuils de population doivent être comparés à la taille de la population nationale, comme pour les autres taxons. Toutefois, dans le cas de taxons menacés à l'échelle mondiale, il est recommandé que la taille de la population du site soit également comparée à la taille de la population mondiale afin de déterminer si la future inscription du taxon sur la Liste rouge de l'UICN permettrait de désigner le site comme une KBA mondiale selon le critère A1. 


\subsubsection{Les espèces qualifiantes selon le critère A1 et ayant un statut différent dans différentes parties de leur aire de répartition}

Si les rangs diffèrent entre les populations reproductrices, non reproductrices et migratrices du taxon (par exemple N1B, N3N, N3M selon le système NatureServe), alors le «rang N» désigne le niveau de menace applicable à la population du site KBA examiné. Par exemple, si le site KBA candidat se trouve dans l'aire de reproduction du taxon, alors dans cet exemple, le «rang $\mathrm{N} »$ de la population reproductrice serait utilisé (N1). Dans ce cas, la population reproductrice totale au Canada serait utilisée pour calculer si le seuil de population est respecté.

\subsection{Utilisation de la taille de la population continentale lorsque des estimations fiables de la population nationale ne sont pas disponibles ou pertinentes (tous les critères)}

L'une des principales différences entre les KBA mondiales et nationales réside dans le dénominateur utilisé pour comparer les estimations de la taille de la population du site (pour les taxons) ou de l'étendue (pour les types d'écosystèmes) afin de vérifier si un seuil de critère est respecté. Dans le cas des évaluations des KBA mondiales selon les critères $\mathrm{A}, \mathrm{B}$ ou $\mathrm{D}$, les estimations du site sont évaluées par rapport aux estimations de la population mondiale afin de calculer le pourcentage de la taille de la population mondiale ou de l'étendue de l'écosystème présent dans un site proposé. En revanche, dans le cas des évaluations des KBA nationales, les données du site sont évaluées par rapport aux estimations nationales de la taille de la population ou de l'étendue de l'écosystème.

Pour certains taxons, cependant, les estimations de la taille de la population au niveau national peuvent ne pas être pratiques ou pertinentes. Dans le cas des espèces qui migrent au-delà des frontières internationales (comme de nombreux taxons d'oiseaux, mais aussi des espèces comme le caribou, les monarques et certaines libellules), les estimations de population nationales peuvent être moins pertinentes que les estimations de population continentales/biogéographiques, sans compter que les estimations de population nationales sont souvent inaccessibles. Dans ces cas, les estimations de la taille de la population continentale sont acceptées pour servir de dénominateur aux évaluations de KBA nationales. En fait, cette décision entraînera l'application de règles plus strictes pour identifier les KBA nationales : une KBA nationale identifiée en fonction de la taille de la population continentale serait également admissible en fonction de la taille de la population nationale, alors que l'inverse peut ne pas être vrai. En pratique, pour ce qui est des espèces qui se déplacent uniquement au Canada à certains moments, la population continentale peut parfois être égale à la population nationale (par exemple, certaines populations de caribous, de nombreuses espèces d'oiseaux migrateurs). 


\section{ANNEXE 2 PROCESSUS D'EXAMEN CANADIEN}

Zones clés pour la biodiversité : Processus d'identification, de délimitation, de sensibilisation et d'examen

Version d'octobre 2020

Le présent document a été préalablement examiné et accepté par le Comité directeur national d'En route vers l'objectif 1 du Canada.

\section{Contexte}

L'objectif principal de l'initiative KBA canadienne, soutenue par l'initiative En route vers l'objectif 1 du Canada, consiste à identifier et à délimiter les sites importants pour la biodiversité au Canada, en appliquant le Standard KBA mondial élaborée par l'UICN pour les KBA mondiales et une adaptation de ces critères pour déterminer les KBA significatives à l'échelle nationale. Notre objectif consiste à présenter des propositions fondées qui intègrent les meilleures connaissances scientifiques et autres disponibles, et à mobiliser les personnes et les groupes susceptibles de posséder des connaissances utiles ou d'avoir des intérêts relatifs à une proposition de KBA. Le processus décrit ci-dessous vise à soutenir l'élaboration de propositions rigoureuses et cohérentes.

Lorsqu'une KBA a été identifiée (c'est-à-dire lorsqu'un lieu particulier répond à un ou plusieurs critères KBA), elle doit être délimitée (c'est-à-dire avec des limites cartographiées). L'identification et la délimitation d'une KBA constituent avant tout un processus scientifique. Les KBA sont identifiées en fonction des éléments de biodiversité présents sur le site et, dans de nombreux cas, elles reflètent le succès des méthodes de gestion existantes. Elles ne requièrent aucune mesure de gestion, mais fournissent plutôt des informations importantes pour permettre d'orienter la conservation de la biodiversité des sites.

Le processus d'examen décrit ci-dessous vise à s'assurer que les KBA sont crédibles sur le plan scientifique. Parallèlement, nous menons des activités de sensibilisation afin que les organismes et les parties prenantes les plus susceptibles d'utiliser les KBA pour éclairer la prise de décision soient informés sur les KBA et en comprennent l'importance.

Étant donné que le processus des KBA au Canada doit tenir compte de la portée vaste et complexe de la biodiversité (espèces, écosystèmes, génétique), ainsi que de la richesse et de la diversité de nos contextes géographiques, sociaux et économiques, nous visons à créer un processus d'identification des KBA qui soit à la fois cohérent et suffisamment souple pour refléter cette diversité.

\section{Étapes du processus d'identification et d'examen des KBA}

\section{Identifier une KBA proposée}

Toute personne ou organisation peut proposer une KBA au Secrétariat KBA canadien. Toutefois, les KBA sont généralement identifiées dans le cadre d'ateliers réunissant des experts spécialisés dans différents taxons (plantes, oiseaux, mammifères, etc.) ou écosystèmes. Il peut s'agir d'experts du gouvernement, d'établissements scientifiques, de groupes autochtones et d'ONG. Cette première étape de l'approche KBA ne nécessite pas nécessairement une contribution exhaustive des experts, mais il faut être raisonnablement certain que des données et des connaissances pertinentes ont été recueillies concernant les espèces, les écosystèmes et d'autres aspects du processus d'évaluation des KBA. Ce processus mené par des experts se limite à la collecte de connaissances et de données pertinentes et à l'interprétation des critères KBA. Durant l'atelier (ou autre processus d'évaluation), les coordonnées des autres personnes possédant des 
connaissances ou des données relatives au site, à l'écosystème ou aux espèces doivent être recueillis afin d'améliorer la rigueur de la proposition de KBA. On pourra communiquer avec ces personnes pour obtenir des renseignements complémentaires et vérifier si le contenu de la proposition est complet et exact.

Le processus d'identification d'une KBA, quel qu'il soit, doit reposer sur les lignes directrices publiées à cet effet et permettre de démontrer clairement comment une zone définie et particulière répond à un ou plusieurs critères KBA. Lorsqu'une personne ou un petit groupe élabore la première ébauche d'une proposition de KBA en l'absence d'un atelier ou d'un autre processus de ce type, il importe de communiquer avec des experts en la matière ainsi qu'avec des détenteurs de connaissances et de veiller à ce que leur contribution soit intégrée dans la proposition. De même, lorsque les ZICO existantes sont évaluées par rapport aux critères KBA, un examen supplémentaire effectué par divers experts garantira que la KBA prend en compte tous les taxons pertinents.

Résultat : Un projet de KBA décrit dans un formulaire de proposition, accompagné de données spatiales concernant les limites de la KBA nominale et l'emplacement des éléments de biodiversité pertinents (espèces ou écosystèmes).

\section{Examen de l'objet}

Une ou plusieurs personnes compétentes doivent examiner la proposition pour vérifier si :

- la base de connaissances de la proposition est suffisante;

- la proposition représente fidèlement les connaissances et les commentaires des experts;

- les critères sont interprétés correctement;

- une limite raisonnable est proposée pour la KBA.

$\mathrm{Si}$ un coordonnateur régional dirige le processus d'identification de la KBA locale, il peut effectuer cette étape, avec l'aide du Secrétariat KBA canadien. Dans les autres cas, le Secrétariat KBA dirigera le processus d'examen.

\section{Examen technique}

La proposition sera examinée par le Secrétariat KBA canadien afin de confirmer qu'elle est complète sur le plan technique et qu'elle répond aux exigences des lignes directrices sur les KBA nationales et mondiales. Le cas échéant, il peut être nécessaire de faire appel à des experts supplémentaires pour combler les lacunes ou les points faibles.

\section{Examen par les détenteurs de droits}

Les gouvernements, les détenteurs de droits autochtones et les propriétaires fonciers (appelés collectivement ici «détenteurs de droits») dont les terres peuvent se trouver dans les limites d'une KBA potentielle (y compris les sites précédemment désignés comme ZICO) seront informés de la délimitation proposée de la KBA et invités à fournir des informations et des commentaires pertinents. Il peut s'agir d'informations que le détenteur des droits choisit de communiquer sur la biodiversité du site ou sur les mesures de conservation appliquées au site, ainsi que sur les propositions d'amélioration des limites de la KBA. Des données nouvelles ou manquantes sur les espèces et les écosystèmes peuvent également être fournies à ce stade, même si cela reste rare lorsque des experts des organismes de gestion ont participé aux premières étapes du processus. Les KBA sont identifiées en fonction de données scientifiques et ne nécessitent pas d'approbation officielle de la part des gouvernements, des détenteurs de droits autochtones ou des propriétaires fonciers. De même, la délimitation d'une KBA ne suppose, ne détermine, ni n'exige aucune mesure de gestion particulière. 


\section{Sensibilisation du public}

Une fois les étapes ci-dessus achevées, le Secrétariat KBA canadien aura normalement :

- un projet de proposition de KBA, au format requis et contenant les informations nécessaires à l'examen par les secrétariats KBA international ou national;

- un résumé destiné au public, comprenant une carte.

S'il y a lieu, le résumé public sera affiché sur le site Web de KBA Canada et des mesures appropriées seront prises pour informer les communautés locales, les propriétaires fonciers et les organisations sur les KBA et leur objectif, ainsi que sur l'identification et la délimitation de certaines KBA susceptibles de les intéresser particulièrement. Bien que cette étape ne soit pas considérée comme faisant partie de l'examen et qu'aucune rétroaction ne soit demandée directement, il sera possible de formuler des commentaires qui seront examinés et pris en considération par le Secrétariat KBA canadien.

\section{Acceptation par le comité de gestion}

Les propositions de KBA achevées sont acceptées par le comité de gestion de KBA Canada et ensuite publiées dans la base de données KBA canadienne. Dans le cas des KBA nationales, aucun autre examen n'est nécessaire, mais les KBA mondiales sont ensuite soumises au Secrétariat KBA mondial.

\section{Approbation des KBA mondiales}

Dans le cas des KBA mondiales, le Secrétariat KBA mondial (situé chez Birdlife International) examinera le formulaire de proposition et la ou les couches spatiales qui l'accompagnent et pourra demander un travail ou un examen supplémentaire si nécessaire. Après l'approbation du Secrétariat KBA mondial, les KBA mondiales seront publiées dans la base de données mondiale des KBA.

\section{Révision périodique}

Les sites doivent être réévalués en fonction des critères et des seuils au moins une fois tous les 8 à 12 ans. Les changements véritables de statut et les changements dans la connaissance du ou des éléments de biodiversité répondant aux critères et aux seuils peuvent tous deux influer sur le statut d'un site comme KBA. Les sites qui ne répondent à aucun critère mondial ou national ne seront plus considérés comme des KBA. 


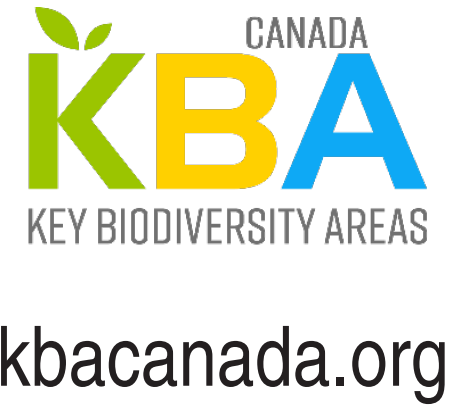

